\title{
Composantes connexes et irréductibles en familles
}

\author{
Matthieu Romagny \\ 14 décembre 2009
}

॥

\begin{abstract}
For an algebraic stack $\mathscr{X}$ flat and of finite presentation over a scheme $S$, we introduce various notions of relative connected components and relative irreducible components. The main distinction between these notions is whether we require the total space of a relative component to be open or closed in $\mathscr{X}$. We study the representability of the associated functors of relative components, and give an application to the moduli stack of curves of genus $g$ admitting an action of a fixed finite group $G$.
\end{abstract}

\section{Introduction}

1.1 Motivation. La preuve de l'irréductibilité de l'espace de modules des courbes de genre $g$ par Deligne et Mumford en 1969 utilise le fait que pour un morphisme $\mathscr{X} \rightarrow S$ propre, plat, de présentation finie, à fibres géométriquement normales, le nombre géométrique de composantes irréductibles des fibres est constant. Dans cette situation comme dans bien d'autres en géométrie algébrique relative, on voudrait en savoir un peu plus sur la variation des composantes connexes et irréductibles dans les fibres d'une famille. Ainsi, dans l'exemple ci-dessus, on peut penser qu'il existe en fait un espace de modules pour les composantes irréductibles relatives (à définir) de l'espace de modules des courbes (lisses, ou stables) de genre $g$ qui est représentable par le schéma $\operatorname{Spec}(\mathbb{Z})$. La contribution principale du présent article est de proposer différentes notions de composantes connexes et irréductibles en familles, de montrer que les foncteurs auxquelles elles donnent naissance sont représentables, de comparer ces notions lorsque c'est possible, et de donner des exemples et contre-exemples. En application, nous étudions les composantes irréductibles du lieu $\mathscr{M}_{g}(G)$ des courbes qui admettent une action d'un groupe fini $G$ fixé.

1.2 Composantes connexes. Il est légèrement plus simple de développer la théorie des composantes connexes; parlons donc d'abord de celles-ci. Au minimum, une composante connexe relative pour $\mathscr{X} \rightarrow S$ doit être un sous-champ $\mathscr{C} \subset \mathscr{X}$ qui est plat et de présentation finie sur $S$, et dont chaque fibre géométrique est une composante connexe de la fibre correspondante de $\mathscr{X}$. Il y a ensuite deux notions naturelles possibles, selon que l'on demande à une telle composante $\mathscr{C}$ d'être ouverte ou fermée dans $\mathscr{X}$.

Comme il s'agit d'une notion de nature essentiellement topologique, on aimerait qu'une composante connexe relative soit déterminée par son support. Ceci mène à considérer en premier lieu des sous-champs ouverts $\mathscr{C} \subset \mathscr{X}$. Si $\mathscr{X}$ est à fibres géométriquement réduites, on montre que le foncteur correspondant $\pi_{0}(\mathscr{X} / S)$ est représentable par un espace algébrique étale et quasi-compact

Mots clé : composantes connexes, composantes irréductibles, critères d'Artin, schéma de Hilbert.

Mathematics Subject Classification : 14A20, 14D06, 14H10, 14D22

Matthieu Romagny, Institut de Mathématiques, Théorie des Nombres, Université Pierre et Marie Curie, Case 82, 4, place Jussieu, F-75252 Paris Cedex 05.

email : romagny@math.jussieu.fr 
(théorème 2.5.2). On obtient même une description très précise de cet espace comme quotient de $\mathscr{X}$ par la relation d'équivalence définie par l'appartenance à la même composante. Malheureusement, dans le cas de fibres non géométriquement réduites, l'effectivité des composantes formelles est prise en défaut et le foncteur des composantes connexes n'est en général pas représentable.

Alternativement, on peut aussi considérer, en guise de composantes relatives, des sous-champs fermés. En s'appuyant sur le schéma de Hilbert, on montre que si $\mathscr{X} \rightarrow S$ est propre, le foncteur correspondant $\pi_{0}(\mathscr{X} / S)^{\mathfrak{f}}$ est représentable par un espace algébrique formel, localement de présentation finie et séparé. Cet espace peut être très (trop) gros, mais le sous-foncteur $\pi_{0}(\mathscr{X} / S)^{\mathfrak{r}}$ des composantes connexes fermées dont les fibres sont géométriquement réduites est représentable par un schéma formel quasi-fini et séparé (théorème 3.2.1).

Lorsque $\mathscr{X} \rightarrow S$ est à la fois à fibres géométriquement réduites et propre, on peut comparer ces constructions. On montre que $\pi_{0}(\mathscr{X} / S), \pi_{0}(\mathscr{X} / S)^{\mathfrak{f}}$ et $\pi_{0}(\mathscr{X} / S)^{\mathfrak{r}}$ sont alors isomorphes, et ils sont aussi isomorphes à la factorisation de Stein de $\mathscr{X}$ (proposition 3.2.5). On peut encore les comparer lorsque $\mathscr{X} \rightarrow S$ est seulement à fibres géométriquement réduites et pur : on trouve que $\pi_{0}(\mathscr{X} / S)^{\mathfrak{f}}$ est un ouvert de $\pi_{0}(\mathscr{X} / S)$.

1.3 Composantes irréductibles. Les questions poséees ci-dessus pour les composantes connexes ont un analogue pour les composantes irréductibles. Le travail auquel cela mène et les réponses obtenues semblent d'ailleurs un peu plus originaux. On définit ainsi un foncteur $\operatorname{Irr}(\mathscr{X} / S)$ de composantes irréductibles ouvertes, qui est représentable par un espace algébrique étale et quasi-compact si $\mathscr{X}$ est à fibres géométriquement réduites (théorème 2.5.2). On définit aussi un foncteur $\operatorname{Irr}(\mathscr{X} / S)^{\mathfrak{f}}$ de composantes irréductibles fermées, mais on montre, en regardant la famille donnée par la conique plane universelle, que même si $\mathscr{X}$ est propre, ce foncteur n'est en général pas représentable par un un espace algébrique formel (voir 3.3.6). Si $\mathscr{X}$ est à fibres géométriquement réduites, ce foncteur est tout de même ouvert dans $\operatorname{Irr}(\mathscr{X} / S)$.

1.4 Application : courbes avec action de $G$. En guise d'application, nous démontrons le résultat suivant. Soit $G$ un groupe fini, $\gamma$ son cardinal, $g \geq 2$ un entier et $\mathscr{M}_{g}(G)$ le sous-champ du champ des courbes projectives lisses de genre $g$ formé des courbes qui admettent une action fidèle de $G$. Alors, sur le schéma de base $S=\operatorname{Spec}(\mathbb{Z}[1 / 30 \gamma])$, le foncteur des composantes irréductibles ouvertes de $\mathscr{M}_{g}(G)$ est représentable par un schéma fini étale (corollaire 3.4.3). Si $G$ n'est pas dans une liste explicite de 10 groupes, ce résultat est même valable sur $\operatorname{Spec}(\mathbb{Z}[1 / 2 \gamma])$. Notons que c'est l'intérêt pour des objets tels que $\mathscr{M}_{g}(G)$ ou d'autres champs classifiants qui justifie l'effort fait pour établir les résultats dans le cadre des champs algébriques.

1.5 Remarques. Dans le cas où $\mathscr{X}$ est un $S$-schéma lisse et quasi-compact, on trouve une brève étude de $\pi_{0}(\mathscr{X} / S)$ dans [LMB], (6.8). Son introduction dans loc. cit. est motivée par l'intérêt pour la notion d'équiconnexité, notion étroitement liée à son tour aux propriétés de séparation de $\pi_{0}(\mathscr{X} / S)$.

De nombreux énoncés classiques de géométrie algébrique connus pour les schémas s'étendent, plus ou moins facilement d'ailleurs, au cadre des champs algébriques. Il est d'usage d'admettre purement et simplement les énoncés dont la preuve est essentiellement la même que pour les schémas, et j'avoue céder parfois à ce travers. Cependant, j'ai préféré vérifier soigneusement que les énoncés de [EGA sur la constructiblité de certaines parties et de certaines propriétés s'adaptent bien aux champs, car ces énoncés sont utilisés abondamment dans ce texte. J'ai donc inclus en fin d'article un premier appendice sur ce sujet, et un second appendice qui utilise le premier pour étendre aux champs certains résultats de [Ro2] sur les schémas purs, utilisés dans le présent article.

1.6 Notation. Dans tout le texte, nous utilisons les notation $(R, K, k, \pi)$ pour désigner un anneau de valuation discrète $R$ de corps de fractions $K$, de corps résiduel $k$, et une uniformisante $\pi$. 
1.7 Remerciements. En premier lieu, je souhaite remercier chaleureusement Pierre Lochak qui m'a posé une question à l'origine de ce travail, en a suivi la progression et a écouté mes réflexions sur la question depuis quelques mois. Je veux aussi remercier Sylvain Brochard, qui m'a fourni l'une des deux preuves données ici d'un énoncé sur les composantes le long d'une section dans le cadre des champs. J'exprime enfin ma gratitude à Angelo Vistoli qui m'a suggéré l'exemple de la conique universelle, et à Sylvain Maugeais pour quelques échanges de courriels sur le thème de l'article.

\section{Table des matières}

1 Introduction

2 Composantes ouvertes

2.1 Définitions et remarques préliminaires . . . . . . . . . . . . 3

2.2 Composantes connexes et irréductibles le long d'une section : énoncés . . . . . . . . 5

2.3 Composantes connexes le long d'une section : preuve . . . . . . . . . . 6

2.4 Composantes irréductibles le long d'une section : preuve . . . . . . . . . . . 8

2.5 Foncteurs des composantes ouvertes . . . . . . . . . . . . . . . 10

2.6 Fonctorialité . . . . . . . . . . . . . . . . . . . . . . . 13

3 Composantes fermées $\quad 14$

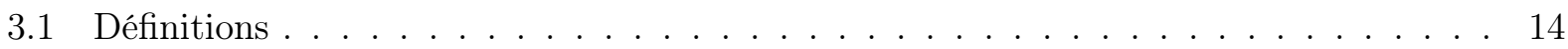

3.2 Représentabilité . . . . . . . . . . . . . . . . . . . . . . 15

3.3 Un contre-exemple . . . . . . . . . . . . . . . . . . . . . . 18

3.4 Exemple : modules des courbes admettant une action . . . . . . . . . . . . . 21

A Propriétés constructibles pour les champs algébriques

B Pureté pour les champs algébriques

\section{Composantes ouvertes}

Une fois définies les composantes ouvertes, la preuve de la représentabilité des foncteurs associés nécessite certains résultats intermédiaires sur les composantes connexes et irréductibles le long d'une section. Ces résultats sont discutés dans 2.2, 2.3 et 2.4. La représentabilité et ses corollaires sont établis dans 2.5 .

\subsection{Définitions et remarques préliminaires}

Une composante connexe relative sera définie comme un sous-champ $\mathscr{C} \subset \mathscr{X}$ plat sur la base et dont les fibres géométriques sont des composantes connexes des fibres correspondantes de $\mathscr{X}$. Comme il s'agit d'une notion essentiellement topologique, on aimerait qu'une composante connexe relative soit déterminée par son support. Ceci mène à considérer des immersions $\mathscr{C} \subset \mathscr{X}$ ouvertes.

Pour un espace topologique possédant un nombre fini de composantes irréductibles, appelons composante irréductible ouverte l'intérieur d'une composante irréductible, ou de manière équivalente, le complémentaire de toutes les composantes irréductibles sauf une. Pour les mêmes raisons que cidessus, il est naturel de souhaiter qu'une composante irréductible relative soit un sous-champ ouvert $\mathscr{I} \subset \mathscr{X}$. Dans les fibres géométriques de $\mathscr{X}$, on est amené à porter l'attention sur les composantes irréductibles ouvertes. On arrive aux définitions suivantes. 
2.1.1 Définitions. Soit $\mathscr{X}$ un champ algébrique de présentation finie sur un schéma $S$.

(1) Une composante connexe ouverte (en abrégé c.c.o.) de $\mathscr{X}$ sur $S$ est un sous-champ ouvert $\mathscr{C} \subset \mathscr{X}$ fidèlement plat et de présentation finie sur $S$, tel que pour tout point géométrique $\bar{s}$ : $\operatorname{Spec}(\Omega) \rightarrow S$, la fibre $\mathscr{C}_{\bar{s}}$ est une composante connexe de $\mathscr{X}_{\bar{s}}$. On note $\pi_{0}(\mathscr{X} / S)$ le foncteur qui à un $S$-schéma $T$ associe l'ensemble des c.c.o. de $\mathscr{X}_{T}$ sur $T$.

(2) Une composante irréductible ouverte (en abrégé c.i.o.) de $\mathscr{X}$ sur $S$ est un sous-champ ouvert $\mathscr{I} \subset \mathscr{X}$ fidèlement plat et de présentation finie sur $S$, tel que pour tout point géométrique $\bar{s}$ : $\operatorname{Spec}(\Omega) \rightarrow S$, la fibre $\mathscr{I}_{\bar{s}}$ est une composante irréductible ouverte de $\mathscr{X}_{\bar{s}}$. On note $\operatorname{Irr}(\mathscr{X} / S)$ le foncteur qui à un $S$-schéma $T$ associe l'ensemble des c.i.o. de $\mathscr{X}_{T}$ sur $T$.

La formation de ces foncteurs commute aux changements de base $S^{\prime} \rightarrow S$.

2.1.2 Lemme. Soit $\mathscr{X}$ un $S$-champ algébrique plat et de présentation finie et soit $F$ l'un des deux foncteurs $\pi_{0}(\mathscr{X} / S)$ ou $\operatorname{Irr}(\mathscr{X} / S)$. Alors, $F$ est un faisceau pour la topologie étale, à diagonale ouverte quasi-compacte. De plus $F$ est étale et quasi-compact sur $S$.

On rappelle que par définition, un faisceau est étale s'il est formellement étale et localement de présentation finie.

Preuve : La propriété de faisceau résulte de faits classiques de théorie de la descente. Pour voir que la diagonale de $F$ est représentable par une immersion ouverte quasi-compacte, il suffit d'observer que si $T$ est un $S$-schéma et $\mathscr{C}, \mathscr{C}^{\prime} \in F(T)$, le lieu des points $t \in T$ tels que $\mathscr{C}_{t}=\mathscr{C}_{t}^{\prime}$ est l'ouvert image de $\mathscr{C} \cap \mathscr{C}^{\prime}$ dans $T$. De plus, utilisant [EGA] IV.8.6.3, on voit que $F$ est localement de présentation finie. Enfin, si $T_{0} \rightarrow T$ une immersion fermée de schémas qui est un homéomorphisme, alors $\mathscr{X} \times{ }_{S} T_{0} \rightarrow \mathscr{X} \times{ }_{S} T$ est un homéomorphisme, de sorte que $F(T) \rightarrow F\left(T_{0}\right)$ est bijectif. Ceci montre que $F$ est formellement étale sur $S$. Il ne reste qu'à montrer que $F$ est quasi-compact sur $S$, ce qui résulte du lemme 2.1 .3 ci-dessous.

2.1.3 Lemme. Sous les mêmes hypothèses que dans 2.1.2, il existe un ouvert $U$ contenant les points maximaux de $S$ tel que la restriction de $F$ à $U$ soit représentable par un $U$-espace algébrique quasi-compact.

On déduit facilement de cet énoncé qu'il existe une stratification $S^{*}=\left\{S_{i}\right\}$ de $S$ telle que $F \times{ }_{S} S^{*}$ est représentable par un $S^{*}$-espace algébrique quasi-compact. En effet, étant donné que $\mathscr{X}$ est de présentation finie sur $S$ et que la formation de $F$ est compatible au changement de base, on peut se ramener au cas où $S$ est noethérien, auquel cas l'assertion provient de 2.1.3 par récurrence noethérienne. Puisque $S^{*} \rightarrow S$ est bijectif, ceci prouve la quasi-compacité annoncée dans 2.1.2.

Preuve : Le raisonnement étant le même pour les deux foncteurs considérés, disons que $F=$ $\pi_{0}(\mathscr{X} / S)$ pour fixer les idées. Soit $\eta$ un point maximal de $S$. D'après l'énoncé sur la diagonale dans le lemme 2.1.2, il suffit de montrer qu'il existe un voisinage ouvert de $\eta$ au-dessus duquel $F$ possède une présentation $P \rightarrow F$, i.e. un morphisme surjectif et fppf (fidèlement plat de présentation finie) depuis un schéma quasi-compact $P$. Comme $F$ est localement de présentation finie, on se ramène au cas où $S$ est affine et de type fini sur $\mathbb{Z}$. Comme $F$ est étale, il revient au même de trouver une

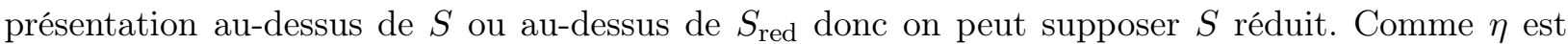
maximal, son anneau local est alors intègre donc quitte à rétrécir $S$, on peut le supposer intègre.

Notons $K$ une extension finie de $k(\eta)$ telle que les sous-schémas fermés réduits des composantes connexes de $\mathscr{X}_{\eta} \otimes K$ soient géométriquement connexes et géométriquement réduits; on peut prendre 
pour $K$ un compositum des corps de définition des composantes connexes réduites de $\mathscr{X}_{\eta} \otimes \overline{k(\eta)}$. Soit $S^{\prime}$ un $S$-schéma affine intègre et de type fini sur $\mathbb{Z}$, de corps de fonctions $K$. Par platitude générique, quitte à restreindre $S$ le morphisme $S^{\prime} \rightarrow S$ est fppf. Alors son image contient un ouvert, donc quitte à restreindre encore $S$ on peut supposer $S^{\prime} \rightarrow S$ surjectif. Il suffit de montrer l'assertion après le changement de base $S^{\prime} \rightarrow S$, on peut donc remplacer $S$ par $S^{\prime}$ et supposer que les composantes connexes $\mathscr{C}_{1, \eta} \ldots, \mathscr{C}_{r, \eta}$ de $\mathscr{X}_{\eta}$ sont géométriquement connexes. Soient $\mathscr{C}_{1}, \ldots, \mathscr{C}_{r}$ des sous-schémas fermés de $\mathscr{X}$ qui induisent les $\mathscr{C}_{i, \eta}$. D'après A.2.2 (iv) et A.2.6 (ii), quitte à rétrécir encore $S$ on peut supposer que les $\mathscr{C}_{i}$ sont les composantes connexes relatives de $\mathscr{X}$. Elles fournissent donc un morphisme surjectif $S \times\{1, \ldots, r\} \rightarrow F$, où à la source la notation désigne la somme disjointe de $r$ copies de $S$. Comme la source et le but sont étales, ce morphisme est étale, et fournit donc une présentation de $\pi_{0}(\mathscr{X} / S)$, qui est donc un espace algébrique quasi-compact au-dessus d'un voisinage de $\eta$.

2.1.4 Proposition. Soit $\mathscr{X}$ un champ algébrique de type fini sur un corps $k$.

(1) Soit $A$ la plus grande sous-k-algèbre séparable de $H^{0}\left(\mathscr{X}, \mathcal{O}_{\mathscr{X}}\right)$. Alors $\pi_{0}(\mathscr{X} / k) \simeq \operatorname{Spec}(A)$.

(2) Soit $B$ la clôture séparable de $k$ dans l'anneau total des fractions de $\mathscr{X}$. Alors $\operatorname{Irr}(\mathscr{X} / k) \simeq$ $\operatorname{Spec}(B)$.

Preuve : (1) D'après le lemme précédent $\pi_{0}(\mathscr{X} / k)$ est représentable par un $k$-espace algébrique étale et quasi-compact, donc par un schéma affine. Par ailleurs, on dispose d'un morphisme $f$ : $\mathscr{X} \rightarrow \operatorname{Spec}(A)$ à fibres géométriquement connexes (voir EGA IV.4.5.15 qui est énoncé pour les schémas mais dont la preuve fonctionne à l'identique pour les champs). Considérons $\mathscr{X}$, vu comme $A$-schéma via $f$, et le morphisme $\mathscr{X} \rightarrow \mathscr{X} \otimes_{k} A$ qui est une immersion ouverte puisque $A$ est étale sur $k$. Ce morphisme fait de $\mathscr{X}$ une c.c.o. de $\mathscr{X} \otimes_{k} A$ sur $\operatorname{Spec}(A)$, ce qui définit un morphisme $g: \operatorname{Spec}(A) \rightarrow \pi_{0}(\mathscr{X} / k)$. Comme $g$ est un isomorphisme après passage à une clôture algébrique de $k$, donc c'est un isomorphisme.

(2) Notons $\mathscr{Y}$ la normalisation de $\mathscr{X}_{\text {red }}$. Nous anticipons un peu sur des résultats de fonctorialité qui seront établis plus tard (voir sous-section [2.6). On a $\operatorname{Irr}(\mathscr{X} / k) \simeq \operatorname{Irr}\left(\mathscr{X}_{\text {red }} / k\right)$, et comme le morphisme de normalisation $\mathscr{Y} \rightarrow \mathscr{X}_{\text {red }}$ est birationnel, on a $\operatorname{Irr}\left(\mathscr{X}_{\text {red }} / k\right) \simeq \operatorname{Irr}(\mathscr{Y} / k)$ (voir corollaire 2.6.2). Par normalité on a $\operatorname{Irr}(\mathscr{Y} / k)=\pi_{0}(\mathscr{Y} / k)$ qui est représentable par le schéma fini, spectre de la plus grande sous- $k$-algèbre séparable de $H^{0}\left(\mathscr{Y}, \mathcal{O}_{\mathscr{Y}}\right)$ (proposition 2.1.4), qui est aussi la plus grande sous- $k$-algèbre séparable du corps de fonctions $k(\mathscr{Y})=k\left(\mathscr{X}_{\text {red }}\right)$. Le résultat en découle.

\subsection{Composantes connexes et irréductibles le long d'une section : énoncés}

Rappelons qu'un point d'un champ $\mathscr{X}$ est une classe d'équivalence de points $x_{K}: \operatorname{Spec}(K) \rightarrow \mathscr{X}$ à valeurs dans un corps $K$, pour la relation qui identifie $x_{K}$ et $x_{L}$ si et seulement s'il existe une extension $M$ de $K$ et $L$ telle que les points $x_{K} \in \mathscr{X}(K)$ et $x_{L} \in \mathscr{X}(L)$ sont isomorphes dans $\mathscr{X}(M)$. On note $x=\left[x_{K}\right]$ le point ainsi défini et $|\mathscr{X}|$ l'espace topologique des points de $\mathscr{X}$. Il y a une bijection entre sous-ensembles ouverts de $|\mathscr{X}|$ et sous-champs ouverts de $\mathscr{X}$, et nous confondrons les deux.

2.2.1 Proposition. Soit $\mathscr{X}$ un $S$-champ algébrique plat, de présentation finie, à fibres géométriquement réduites et soit $g: S \rightarrow \mathscr{X}$ une section. Pour tout $s \in S$, on note $\mathscr{C}_{s}$ la composante connexe de $g(s)$ dans $\mathscr{X}_{s}$. Alors, la réunion des $\left|\mathscr{C}_{s}\right|$ est un ouvert $C \subset|\mathscr{X}|$, correspondant à un souschamp ouvert $\mathscr{C} \subset \mathscr{X}$, de présentation finie sur $S$, et dont la formation commute au changement de base. 
Nous appellerons $\mathscr{C}$ la c.c.o. de $\mathscr{X}$ le long de la section $g$. S'il est utile de préciser la section, nous noterons $\mathscr{C}_{s}(g)$ au lieu de $\mathscr{C}_{s}$ et $\mathscr{C}(g)$ au lieu de $\mathscr{C}$.

2.2.2 Définition. Soit $X$ un espace topologique. On appelle lieu unicomposante de $X$ l'ensemble des points de $X$ qui n'appartiennent qu'à une composante irréductible de $X$.

Soit $\mathscr{X}$ un $S$-champ algébrique et $x$ un point de l'espace topologique $|\mathscr{X}|$. Notons $s$ l'image de $x$ dans $S$ et désignons par un indice $(\cdot)_{\bar{s}}$ le changement de base au spectre d'une clôture algébrique du corps résiduel de $s$. Il est facile de voir que la propriété pour $x_{K, \bar{s}}: \operatorname{Spec}(K)_{\bar{s}} \rightarrow \mathscr{X}_{\bar{s}}$ de se factoriser par le lieu unicomposante de la fibre géométrique $\mathscr{X}_{\bar{s}}$ est indépendante du représentant $x_{K}: \operatorname{Spec}(K) \rightarrow \mathscr{X}$ choisi pour $x$. Ceci justifie la définition suivante.

2.2.3 Définition. Soit $\mathscr{X}$ un $S$-champ algébrique. On appelle lieu unicomposante (relatif) de $\mathscr{X}$ sur $S$ l'ensemble des points $x \in|\mathscr{X}|$, d'image $s$ dans $S$, tels que $x_{K, \bar{s}}: \operatorname{Spec}(K)_{\bar{s}} \rightarrow \mathscr{X}_{\bar{s}}$ se factorise par le lieu unicomposante de $\mathscr{X}_{\bar{s}}$, pour un (ou, de manière équivalente, n'importe quel) représentant $x_{K}: \operatorname{Spec}(K) \rightarrow \mathscr{X}$.

Noter que si $\mathscr{X}$ est un champ sur un corps $k$, l'inclusion du lieu unicomposante (relatif) de $\mathscr{X}$ sur $k$ dans le lieu unicomposante (absolu) de $|\mathscr{X}|$ est en général stricte. C'est le cas par exemple pour la conique sur $\mathbb{Q}$ d'équation $x^{2}-2 y^{2}=0$.

2.2.4 Proposition. Soit $\mathscr{X}$ un $S$-champ algébrique de présentation finie, à fibres géométriquement réduites.

(i) Le lieu unicomposante de $\mathscr{X}$ sur $S$ est un ouvert $U \subset|\mathscr{X}|$, correspondant à un sous-champ ouvert $S$-dense $\mathscr{U} \subset \mathscr{X}$, de présentation finie sur $S$, dont la formation commute au changement de base.

(ii) Soit $g: S \rightarrow \mathscr{X}$ une section à valeurs dans $\mathscr{U}$. Pour tout $s \in S$, on note $\mathscr{I}_{s}$ la composante irréductible ouverte de $g(s)$ dans $\mathscr{X}_{s}$. Alors, la réunion des $\left|\mathscr{I}_{s}\right|$ est un ouvert $I \subset|\mathscr{X}|$, correspondant à un sous-champ ouvert $\mathscr{I} \subset \mathscr{X}$, de présentation finie sur $S$, dont la formation commute au changement de base.

Nous appellerons $\mathscr{I}$ la c.i.o. de $\mathscr{X}$ le long de la section $g$. S'il est utile de préciser la section, nous noterons $\mathscr{I}_{s}(g)$ au lieu de $\mathscr{I}_{s}$ et $\mathscr{I}(g)$ au lieu de $\mathscr{I}$.

2.2.5 Remarque. Le lemme 2.2.1 est faux en général sans hypothèse de platitude. Pour un contre-exemple, prenons un anneau de valuation discrète $(R, K, k, \pi)$ et le schéma affine d'anneau $R[x, y] /(x y(y-1), \pi y(y-1))$ muni de n'importe quelle $R$-section adhérence d'un point $K$-rationnel de la fibre générique. Il s'agit simplement du schéma obtenu à partir du $R$-schéma plat réunion de trois droites $x y(y-1)=0$, en enlevant de la fibre générique la composante irréductible ouverte centrale d'équation $x=0$.

Il est remarquable qu'au contraire, la platitude n'est pas nécessaire pour le lemme 2.2.4.

\subsection{Composantes connexes le long d'une section : preuve}

Dans cette section, nous démontrons la proposition 2.2.1. Nous démontrons d'abord que la formation de la composante connexe le long de la section $g$ commute au changement de base. Ceci est conséquence du lemme suivant : 
2.3.1 Lemme. Soient $k$ un corps et $f: \mathscr{Y} \rightarrow \mathscr{X}$ un morphisme de $k$-champs algébriques. On suppose que $\mathscr{X}$ est connexe et $\mathscr{Y}$ est géométriquement connexe. Alors, $\mathscr{X}$ est géométriquement connexe.

Preuve : La preuve de [EGA] IV.4.5.13 fonctionne à l'identique pour les champs algébriques.

Pour tout morphisme de changement de base $S^{\prime} \rightarrow S$, notons $\mathscr{X}^{\prime}=\mathscr{X} \times_{S} S^{\prime}, g^{\prime}: S^{\prime} \rightarrow \mathscr{X}^{\prime}$ la section déduite de la section $g$, et $C^{\prime}$ la réunion des $\left|\mathscr{C}_{s^{\prime}}\left(g^{\prime}\right)\right|$. Le fait que la formation de $\mathscr{C}$ commute au changement de base sera conséquence du fait que $C^{\prime}$ est la préimage de $C$ par l'application continue $\left|\mathscr{X}^{\prime}\right| \rightarrow|\mathscr{X}|$. Ceci peut être vérifié fibre à fibre. Or, si $s^{\prime} \in S^{\prime}$ a pour image $s \in S$, la composante connexe $\mathscr{C}_{s}$ est géométriquement connexe d'après le lemme 2.3.1 appliqué à la section $g$. Il s'ensuit que $\mathscr{C}_{s^{\prime}}^{\prime}=\mathscr{C}_{s} \otimes_{k(s)} k\left(s^{\prime}\right)$, d'où notre assertion.

Il reste à démontrer que $C$ est un ouvert. Ce résultat est démontré pour les schémas dans EGA IV.15.6.5. Nous donnerons deux preuves de son extension au cas des champs algébriques. La première m'a été communiquée par Sylvain Brochard et procède par réduction au cas des schémas. La deuxième suit directement la preuve de [EGA] pour les schémas; sa raison d'être est que, comme nous l'expliquons dans 2.4.1, elle s'adaptera mieux que la première au cas des composantes irréductibles le long d'une section, et elle servira alors de modèle.

2.3.2 Première preuve. On procède par réduction au cas des schémas, avec les méthodes du paragraphe 4.2 et notamment du lemme 4.2 .8 de [Bro]. Dans loc. cit., la notation pour $\mathscr{C}$ est $X^{0}$ et pour faciliter la transcription, nous adopterons cette notation dans le reste de 2.3.2. On note $X=|\mathscr{X}|$ et on adopte les notations du diagramme suivant :

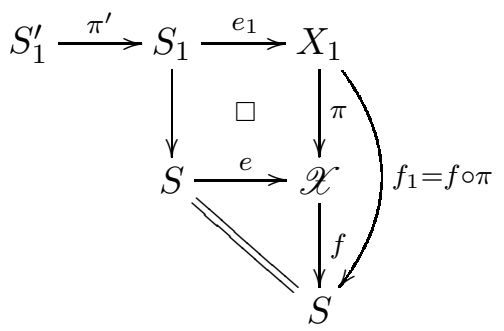

dans lequel $\pi: X_{1} \rightarrow \mathscr{X}$ est une présentation de $\mathscr{X}$ par un schéma et $\pi^{\prime}: S_{1}^{\prime} \rightarrow S_{1}$ est une présentation de l'espace algébrique $S_{1}:=S \times \mathscr{X} X_{1}$ par un schéma. On considère le diagramme de schémas :

$$
S_{1}^{\prime} \stackrel{e_{1} \circ \pi^{\prime}}{\longrightarrow} X_{1} \stackrel{f_{1}}{\longrightarrow} S .
$$

On note $W_{0}:=X_{1}^{0}\left(e_{1} \circ \pi^{\prime}\right)$ (cf. lemme 4.2.7 de [Bro]) le sous-ensemble de $X_{1}$ dont la fibre au-dessus d'un point $s \in S$ est la réunion des composantes connexes de $\left(X_{1}\right)_{s}$ qui rencontrent $e_{1} \circ \pi^{\prime}\left(S_{1}^{\prime}\right)$. Comme l'image continue d'un connexe est connexe, on voit que $\pi\left(W_{0}\right)$ est inclus dans $X^{0}$. Vu que $\pi$ est lisse, on en déduit que $f_{1}$ est universellement ouvert en tout point de $W_{0}$. Par [Bro], 4.2 .7 (ii) a) il en résulte que $W_{0}$ est un ouvert de $X_{1}$.

On considère maintenant $V_{1}=\pi^{-1}\left(\pi\left(W_{0}\right)\right)$. C'est un ouvert de $X_{1}$ qui contient $W_{0}$ (c'est le saturé de $W_{0}$ pour la relation d'équivalence définie par $\pi$ ). D'après [Bro], 4.2 .7 (ii) b) appliqué au diagramme

$$
V_{1} \stackrel{e_{1} \circ \pi^{\prime}}{\longrightarrow} X_{1} \stackrel{f_{1}}{\longrightarrow} S
$$

on voit que $W_{1}:=X_{1}^{0}\left(V_{1} \hookrightarrow X_{1}\right)$ est un ouvert de $X_{1}$. On poursuit le processus en posant successivement

$$
V_{2}=\pi^{-1}\left(\pi\left(W_{1}\right)\right) ; W_{2}=X_{1}^{0}\left(V_{2} \hookrightarrow X_{1}\right) ; \ldots ; V_{n}=\pi^{-1}\left(\pi\left(W_{n-1}\right)\right) ; W_{n}=X_{1}^{0}\left(V_{n} \hookrightarrow X_{1}\right) \ldots
$$


À chaque fois, on a $\pi\left(W_{i}\right) \subset X^{0}$ si bien que $f_{1}$ est universellement ouvert en tout point de $W_{i}$ et le lemme 4.2 .7 (ii) b) de [Bro] permet d'affirmer que $W_{i}$ est ouvert. On obtient ainsi une suite croissante d'ouverts de $X_{1}$ :

$$
W_{0} \subset V_{1} \subset W_{1} \subset V_{2} \subset W_{2} \subset \cdots
$$

On note $V=\cup_{i} V_{i}=\cup_{i} W_{i}$. Pour conclure, il suffit de montrer que $\pi(V)=X^{0}$. On peut pour cela raisonner fibre par fibre et supposer que $S$ est le spectre d'un corps. Quitte à remplacer $X$ par $X^{0}$, on peut aussi supposer que $X$ est connexe (c'est-à-dire que $X^{0}=X$ ). Vu la construction des $V_{i}$ (resp. des $W_{i}$ ) il est clair que $V$ est une réunion de fibres de $\pi$ (resp. de composantes connexes de $\left.X_{1}\right)$.

Pour conclure, il suffit de montrer que $\pi(V)$ contient toutes les composantes irréductibles qu'il rencontre, car il sera alors fermé, ouvert et non vide dans $X$ connexe. Soient donc $x \in \pi(V)$ et $Z$ une composante irréductible de $X$ qui contient $x$. Soit $z \in Z$, il faut montrer que $z \in \pi(V)$. Soit $U$ un ouvert connexe de $X_{1}$ dont l'image contient $z$. Comme $\pi(V) \cap Z$ et $\pi(U) \cap Z$ sont deux ouverts non vides de $Z$, leur intersection est non vide. Comme par ailleurs $V$ est une réunion de fibres de $\pi$, on en déduit que $U \cap V$ est non vide. Enfin, vu que $U$ est connexe et que $V$ est une réunion de composantes connexes de $X_{1}$ on voit que $U$ est inclus dans $V$, si bien que $z \in \pi(V)$. Ceci conclut la preuve de 2.2 .1 .

2.3.3 Deuxième preuve. Revenons aux notations $\mathscr{C}(g), C(g)$ pour désigner les composantes connexes le long d'une section. Nous suivons la preuve de [EGA] IV.15.6.5, en soulignant les modifications nécessaires pour passer aux champs algébriques. Comme la formation de $C$ commute au changement de base, en particulier on peut localiser et supposer $S=\operatorname{Spec}(A)$ affine. Par les arguments habituels, comme $\mathscr{X}$ est de présentation finie sur $S$, on se ramène au cas où $A$ est noethérien.

On montre ensuite que $C$ est localement constructible. Les arguments de EGA IV.9.7.12 restent valables, avec quelques modifications mineures pour adapter aux champs algébriques les lemmes nécessaires de [EGA] IV.9 sur la constructiblité. Ces modifications sont indiquées dans l'annexe @, voir notamment A.2.2 (i), A.2.2 (iv) et A.2.6 (ii).

Il reste à montrer que $C$ est stable par générisation. Pour cela, on est ramené au cas où $S$ est le spectre d'un anneau de valuation discrète $R=(R, K, k, \pi)$, et on peut supposer $R$ complet et $k$ algébriquement clos. On peut enlever de $\mathscr{X}$ les composantes connexes de $\mathscr{X}_{k}$ qui ne rencontrent pas $g(S)$, fermées dans $\mathscr{X}$, et donc supposer $\mathscr{X}_{k}$ connexe. On peut ensuite remplacer $\mathscr{X}$ par sa composante connexe contenant $g(S)$ et donc supposer $\mathscr{X}$ connexe. Il suit alors du lemme B.2 que $\mathscr{X}_{K}$ est connexe, donc $C=|\mathscr{X}|$ est ouvert, ce qui termine la preuve.

\subsection{Composantes irréductibles le long d'une section : preuve}

Dans cette section, nous démontrons la proposition 2.2.4.

2.4.1 Remarques préliminaires. Il est naturel d'essayer de prouver la proposition 2.2.4 en deux étapes, d'abord dans le cas où $\mathscr{X}$ est un schéma, puis en ramenant le cas général à ce cas en utilisant un atlas et diverses techniques semblables à celles de 2.3.2. Ces méthodes font jouer un rôle important à des parties $X^{0}(e)$, réunions de composantes connexes de $X_{s}$ qui rencontrent $e(V)$, où $e: V \rightarrow \mathscr{X}$ est un $S$-morphisme depuis un $S$-schéma $V$ distinct de $S$, typiquement un morphisme lisse surjectif issu d'un atlas de $\mathscr{X}$. On est amené à considérer $X^{1}(e)$, réunion des composantes irréductibles ouvertes de $X_{s}$ qui rencontrent $e(V)$, pour des $e: V \rightarrow \mathscr{X}$ à valeurs dans le lieu unicomposante. On rencontre alors les problèmes suivants : 
(i) On ne peut pas toujours relever des points du lieu unicomposante en des points du lieu unicomposante, même par des morphismes finis étales. Un exemple est donné par le revêtement double non trivial de la cubique nodale (voir figure 1).
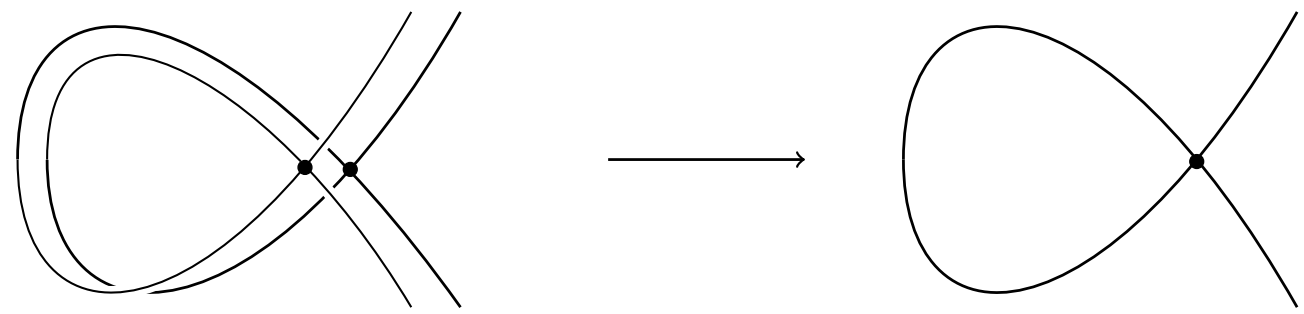

Figure 1 - Revêtement double de la cubique nodale

(ii) La formation de $X^{1}(e)$ ne commute pas au changement de base. Ceci est lié au fait que la formation des composantes irréductibles ouvertes sans point rationnel ne commute pas au changement de base. Ce phénomène se produit même si $V \rightarrow S$ est fini étale : prendre pour $X$ la conique sur $S=\operatorname{Spec}(\mathbb{Q})$ d'équation $x^{2}-2 y^{2}=0$, et $e: \operatorname{Spec}(\mathbb{Q}(\sqrt{2}) \rightarrow X$ donné par le point $(x, y)=(\sqrt{2}, 1)$.

À cause de ces difficultés, nous ne présentons pas de preuve de la proposition 2.2 .4 analogue à 2.3 .2 ,

2.4.2 Preuve de 2.2.4. Nous démontrons maintenant 2.2.4 en suivant une stratégie classique, comme dans 2.3.3. Le fait que la formation du lieu unicomposante relatif commute au changement de base est une conséquence directe de sa définition. Pour vérifier la propriété analogue pour la composante irréductible ouverte le long d'une section, nous utiliserons le lemme suivant, qui est une variante de [EGA] IV.4.5.13.

2.4.3 Lemme. Soient $k$ un corps et $f: \mathscr{Y} \rightarrow \mathscr{X}$ un morphisme de $k$-champs algébriques. On suppose que $\mathscr{X}$ est irréductible et ne possède qu'un nombre fini de composantes irréductibles géométriques, que $\mathscr{Y}$ est géométriquement irréductible et que la préimage par $f$ du lieu unicomposante de $\mathscr{X} / k$ est non vide. Alors, $\mathscr{X}$ est géométriquement irréductible.

Preuve : Soit $k^{\prime}$ une clôture algébrique de $k$; on note avec un ${ }^{\prime}{ }^{\prime} »$ toutes les données obtenues par changement de base de $k$ à $k^{\prime}$, dont le morphisme $f^{\prime}: \mathscr{Y}^{\prime} \rightarrow \mathscr{X}^{\prime}$. Soit $p: \mathscr{X}^{\prime} \rightarrow \mathscr{X}$ la projection, qui est ouverte et fermée. Obervons que le lieu unicomposante $\mathscr{U}$ de $\mathscr{X} / k$ est un sous-champ ouvert, puisque c'est l'image par $p$ du lieu unicomposante de $\mathscr{X}^{\prime} / k^{\prime}$ qui est ouvert. La démonstration ne fait intervenir que les espaces topologiques sous-jacents à $\mathscr{X}, \mathscr{Y}, \mathscr{U}$ et à leurs homologues sur $k^{\prime}$; on les note $X, Y, U$, etc. Si $X^{\prime}$ n'est pas irréductible, on peut partager ses composantes irréductibles en deux paquets disjoints et former les fermés $F^{\prime}, G^{\prime}$ réunions des composantes irréductibles de chacun des deux paquets, et les ouverts disjoints $A^{\prime}=X^{\prime} \backslash G^{\prime}, B^{\prime}=X^{\prime} \backslash F^{\prime}$. On note $F, G, A, B$ les images dans $X$. Puisque $p$ est ouverte et fermée, on voit que $A$ et $B$ sont des ouverts denses de $X$ et que $F$ et $G$ sont des fermés d'intérieur non vide, donc $F=G=X$. De plus, comme $p\left(X^{\prime} \backslash U^{\prime}\right) \subset X \backslash U$ on trouve $U \subset A$ et $U \subset B$. Puisque par hypothèse $f^{-1}(U)$ est non vide, il découle de ce qui précède que les ouverts disjoints $\left(f^{\prime}\right)^{-1}\left(A^{\prime}\right)$ et $\left(f^{\prime}\right)^{-1}\left(B^{\prime}\right)$ sont non vides. Ceci n'est pas possible, car $Y^{\prime}$ est supposé irréductible.

Soient $S^{\prime} \rightarrow S$ un morphisme, $\mathscr{X}^{\prime}=\mathscr{X} \times{ }_{S} S^{\prime}, g^{\prime}: S^{\prime} \rightarrow \mathscr{X}^{\prime}$ la section déduite de la section $g$, et $I^{\prime}$ la réunion des $\left|\mathscr{I}_{s^{\prime}}\left(g^{\prime}\right)\right|$. Vérifions que la partie $I^{\prime}$ est la préimage de $I$ par l'application continue 
$\left|\mathscr{X}^{\prime}\right| \rightarrow|\mathscr{X}|$. On le vérifie fibre à fibre. Si $s^{\prime} \in S^{\prime}$ a pour image $s \in S$, la composante irréductible ouverte $\mathscr{I}_{s}$ est géométriquement irréductible d'après le lemme 2.3.1 appliqué à la section $g$. Utilisant la caractérisation des composantes irréductibles ouvertes comme ouverts irréductibles maximaux et le fait que le morphisme $\mathscr{X}_{s^{\prime}}^{\prime} \rightarrow \mathscr{X}_{s}$ est ouvert, on voit que $\mathscr{I}_{s^{\prime}}^{\prime}=\mathscr{I}_{s} \otimes_{k(s)} k\left(s^{\prime}\right)$. L'assertion à démontrer en découle.

Il nous reste à démontrer que $U$ et $I$ sont ouverts, ce que nous ferons en même temps. Comme la formation de $U$ et $I$ commute au changement de base, on peut travailler étale-localement sur $S$. En particulier, on peut supposer que $S=\operatorname{Spec}(A)$ est affine. Comme $\mathscr{X} \rightarrow S$ est de présentation finie, on peut ensuite supposer que $A$ est noethérien.

Montrons que $U$ et $I$ sont localement constructibles. On se ramène immédiatement au cas où $S$ est intègre de point générique $\eta$. Soient $\mathscr{Z}_{0, \eta}, \ldots, \mathscr{Z}_{d, \eta}$ les composantes irréductibles de $\mathscr{X}_{\eta}$, en supposant que $g(\eta) \in \mathscr{Z}_{0, \eta}$ dans le cas ii). Quitte à faire une extension étale de $S$, on peut supposer que $\mathscr{Z}_{i, \eta}$ est géométriquement intègre pour tout $i$. Soit $\mathscr{Z}_{i}$ un sous-champ fermé de $\mathscr{X}$ dont la fibre au point $\eta$ est $\mathscr{Z}_{i, \eta}$. D'après A.2.2 (i), (ii), (iv) et A.2.6 (iv), quitte à remplacer $S$ par un voisinage de $\eta$, on peut supposer que les $\mathscr{Z}_{i}$ recouvrent $\mathscr{X}$ et que pour tout $s \in S$, leurs fibres sont des fermés géométriquement intègres tels que $\mathscr{Z}_{i, s} \not \subset \mathscr{Z}_{j, s}$ pour $i \neq j$. Par ailleurs dans le cas ii) on a $g^{-1}\left(\mathscr{Z}_{0}\right)=S$ car ce fermé contient le point générique de $S$ qui est irréductible, et $g(S)$ est inclus dans le complémentaire dans $\mathscr{X}$ de $\cup_{i \neq 0} \mathscr{Z}_{i}$. Il est alors clair que $U$ est égal au complémentaire de $\cup_{i, j}\left|\mathscr{Z}_{i} \cap \mathscr{Z}_{j}\right|$ et que $I$ est égal au complémentaire de $\cup_{i \neq 0}\left|\mathscr{Z}_{i}\right|$, qui sont des parties ouvertes, donc localement constructibles.

On conclut enfin que $U$ et $I$ sont ouverts. D'après ce qui précède, on peut supposer que $S$ est le spectre d'un anneau de valuation discrète $(R, K, k, \pi)$ que l'on peut supposer complet à corps résiduel algébriquement clos. On peut aussi enlever de $\mathscr{X}$ ses composantes irréductibles incluses dans $\mathscr{X}_{k}$, puisqu'elles sont fermées et ne rencontrent pas $\mathscr{I}_{k}$, et donc supposer $\mathscr{X}$ plat sur $S$. Il suffit de montrer que $U$ et $I$ sont ouverts au voisinage d'une quelconque composante irréductible

$\mathscr{Y}$ de $\mathscr{X}_{k}$, que l'on choisit comme étant la composante contenant $g(\operatorname{Spec}(k))$ dans le cas ii). On peut donc enlever de $\mathscr{X}$ toutes les composantes irréductibles de $\mathscr{X}_{k}$ distinctes de $\mathscr{Y}$ et supposer $\mathscr{X}_{k}$ géométriquement intègre. On peut ensuite enlever les composantes irréductibles de $\mathscr{X}$ incluses dans $\mathscr{X}_{K}$ et donc supposer $\mathscr{X}$ pur sur $S$ (pour des détails sur la pureté, voir l'annexe B, [RG] ou [Ro2]). Il suit alors du th. B.4 (iii) que $\mathscr{X}_{K}$ est intègre, de sorte que $U=I=|\mathscr{X}|$ et notre assertion est prouvée.

\subsection{Foncteurs des composantes ouvertes}

Nous allons démontrer que pour les champs plats, de présentation finie et à fibres géométriquement réduites, les foncteurs $\pi_{0}(\mathscr{X} / S)$ et $\operatorname{Irr}(\mathscr{X} / S)$ sont représentables par des espaces algébriques étales et quasi-compacts. Nous précisons tout d'abord un petit point de théorie (2.5.1) avant d'énoncer le théorème (2.5.2).

2.5.1 Quotient d'un champ algébrique par un groupoïde. Nous aurons besoin ci-dessous de considérer le quotient d'un champ algébrique par une relation d'équivalence étale. Cette opération ne présente pas véritablement de difficulté, et les spécialistes de l'homotopie savent même faire beaucoup mieux (voir notamment [TV], paragraphe 1.3.4). Malheureusement, leurs travaux restent difficiles à lire pour de nombreux géomètres, y compris par le rédacteur de ces lignes. Faute de référence dans laquelle ces résultats sont énoncés dans un cadre plus simple, adapté à nos besoins, nous esquisserons ces constructions.

Soit $S$ un schéma. Nous appellerons groupoïde plat (en champs algébriques) et nous noterons $\mathscr{X}_{1} \rightrightarrows \mathscr{X}_{0}$ la donnée de deux $S$-champs algébriques $\mathscr{X}_{0}, \mathscr{X}_{1}$ et de : 
(1) deux morphismes fidèlement plats et de présentation finie $s, t: \mathscr{X}_{1} \rightarrow \mathscr{X}_{0}$,

(2) un morphisme de composition $c: \mathscr{X}_{1} \times_{t, \mathscr{X}_{0}, s} \mathscr{X}_{1} \rightarrow \mathscr{X}_{1}$,

(3) un morphisme d'identité $e: \mathscr{X}_{0} \rightarrow \mathscr{X}_{1}$,

(4) un morphisme d'inversion $i: \mathscr{X}_{1} \rightarrow \mathscr{X}_{1}$,

ainsi que d'un certain nombre de 2-isomorphismes de compatibilité exprimant la 2-commutativité des diagrammes des axiomes bien connus de groupoïde (voir par exemple [LMB], 2.4.3). Par souci de légèreté, nous ne précisons pas ces 2-isomorphismes, mais ils peuvent facilement être écrits en s'inspirant par exemple de [Ro1], section 1. Comme dans la definition 1.3 de [Ro1], on a une notion de groupoïde strict correspondant au cas où tous ces 2-isomorphismes sont l'identité. Étant donné un champ $\mathscr{Y}$, un morphisme $\mathscr{X}_{1}$-invariant de $\mathscr{X}_{0}$ vers $\mathscr{Y}$ consiste en la donnée d'un morphisme $f: \mathscr{X}_{0} \rightarrow \mathscr{Y}$ et d'un 2-isomorphisme $\sigma: f \circ s \Rightarrow f \circ t$ compatible avec les 2-isomorphismes de structure du groupoïde. On peut former le stabilisateur $\mathscr{S}$ défini par le 2-produit fibré

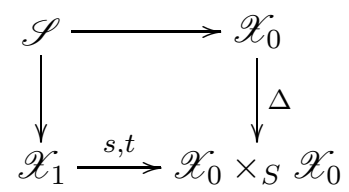

où $\Delta$ est la diagonale. L'énoncé de représentabilité qui nous intéresse est que si $\mathscr{S} \rightarrow \mathscr{X}_{0}$ est un morphisme représentable, il existe un champ algébrique quotient $\pi: \mathscr{X}_{0} \rightarrow \mathscr{X}:=\mathscr{X}_{0} / \mathscr{X}_{1}$ qui vérifie la 2-propriété universelle suivante : pour tout morphisme de champs algébriques $f: \mathscr{X}_{0} \rightarrow \mathscr{Y}$ qui est $\mathscr{X}_{1}$-invariant, il existe un morphisme $f^{\prime}: \mathscr{X} \rightarrow \mathscr{Y}$ tel que $f=f^{\prime} \circ \pi$, unique à un unique 2-isomorphisme près.

On peut décrire $\mathscr{X}_{0} / \mathscr{X}_{1}$ de la manière suivante : c'est le champ associé à la catégorie fibrée en groupoïdes $\mathscr{P}$ dont les fibres sont les catégories $\mathscr{P}(T)$, pour des $S$-schéma $T$ variables, telles que $\operatorname{Obj}(\mathscr{P}(T))=\mathscr{X}_{0}(T)$ et $\operatorname{Hom}_{\mathscr{P}(T)}(x, y)$ est l'ensemble des paires $\left(x_{1}, \varphi\right)$ où $x_{1} \in \mathscr{X}_{1}(T)$ vérifie $s\left(x_{1}\right)=x$ et $\varphi: t\left(x_{1}\right) \rightarrow y$ est un morphisme dans $\mathscr{X}_{0}(T)$. La composition des morphismes dans $\mathscr{P}(T)$ se fait ainsi : étant donnés un morphisme $\left(x_{1}, \varphi\right)$ entre $x$ et $y$ et un morphisme $\left(y_{1}, \psi\right)$ entre $y$ et $z$, le triplet $\left(x_{1}, y_{1}, \varphi\right)$ est un objet de $\mathscr{X}_{1} \times_{t, \mathscr{X}_{0}, s} \mathscr{X}_{1}$ et on peut poser $x_{1}^{\prime}:=c\left(x_{1}, y_{1}, \varphi\right)$. Le composé de $\left(x_{1}, \varphi\right)$ et $\left(y_{1}, \psi\right)$ est alors $\left(x_{1}^{\prime}, \psi\right)$.

Le résultat de quotient que nous venons d'esquisser recouvre le quotient d'un champ algébrique par l'action d'un schéma en groupes comme dans [Ro1, par l'action libre d'un champ algébrique en groupes comme dans $[\mathrm{La}]$, ou par une relation d'équivalence plate, comme ci-dessous.

2.5.2 Théorème. Soit $\mathscr{X}$ un $S$-champ algébrique plat, de présentation finie, à fibres géométriquement réduites.

(i) Les foncteurs $\pi_{0}(\mathscr{X} / S)$ et $\operatorname{Irr}(\mathscr{X} / S)$ sont représentables par des espaces algébriques étales et quasi-compacts sur $S$.

(ii) Soit la relation d'équivalence définie comme sous-catégorie pleine $\mathscr{R} \subset \mathscr{X} \times \mathscr{X}$ telle que deux points $u, v: T \rightarrow \mathscr{X}$ sont équivalents ssi pour tout point géométrique $t: \operatorname{Spec}(\Omega) \rightarrow T$, les points $u(t)$ et $v(t)$ sont dans la même composante connexe de $\mathscr{X}_{\Omega}$. Cette relation est représentable par la c.c.o. de $\mathscr{X} \times \mathscr{X}$ le long de la section diagonale. De plus, il existe un morphisme $\mathscr{X} \rightarrow \pi_{0}(\mathscr{X} / S)$ grâce auquel $\mathscr{X}$ s'identifie à la c.c.o. universelle et $\pi_{0}(\mathscr{X} / S)$ au quotient $\mathscr{X} / \mathscr{R}$.

(iii) Soit $\mathscr{U} \subset \mathscr{X}$ le lieu unicomposante de $\mathscr{X}$ sur $S$, et soit la relation d'équivalence $\mathscr{S} \subset \mathscr{U} \times$ $\mathscr{U}$, sous-catégorie pleine telle que deux points $u, v: T \rightarrow \mathscr{U}$ sont équivalents ssi pour tout point géométrique $t: \operatorname{Spec}(\Omega) \rightarrow T$, les points $u(t)$ et $v(t)$ sont dans la même composante irréductible 
ouverte de $\mathscr{U}_{\Omega}$. Cette relation est représentable par la c.i.o. de $\mathscr{U} \times \mathscr{U}$ le long de la section diagonale. De plus, il existe un morphisme $\mathscr{U} \rightarrow \operatorname{Irr}(\mathscr{X} / S)$ grâce auquel $\mathscr{U}$ s'identifie à la c.i.o. universelle et $\operatorname{Irr}(\mathscr{X} / S)$ au quotient $\mathscr{U} / \mathscr{S}$.

Rappelons que le lieu unicomposante $\mathscr{U}$ est un sous-champ ouvert de $\mathscr{X}$, voir la proposition 2.2.4.

Preuve : (i) On utilise les critères de représentabilité d'Artin (corollaire 5.2 de [Ar1]) pour les foncteurs $F=\pi_{0}(\mathscr{X} / S)$ et $F=\operatorname{Irr}(\mathscr{X} / S)$. Le raisonnement étant le même pour les deux foncteurs considérés, disons que $F=\pi_{0}(\mathscr{X} / S)$ pour fixer les idées. On se ramène par les arguments habituels au cas où $S$ est le spectre d'une $\mathbb{Z}$-algèbre de type fini. D'après le lemme 2.1.2, le foncteur $F$ est un faisceau localement de présentation finie à diagonale ouverte (condition (1) d'Artin) et de plus formellement étale sur $S$, donc les théories de déformations et d'obstructions sont nulles (conditions (2) et (4) d'Artin).

Il ne reste que la condition (3) d'effectivité des composantes formelles à vérifier : on doit montrer que pour tout anneau local complet $(R, m)$ et tout morphisme $\operatorname{Spec}(R) \rightarrow S$ qui induit une extension de corps résiduels de type fini, l'application $F(R) \rightarrow \lim F\left(R / m^{n}\right)$ est injective et d'image dense. Puisque les applications $F\left(R / m^{n+1}\right) \rightarrow F\left(R / m^{n}\right)$ sont des bijections, il s'agit juste de montrer que l'application $\varphi: F(R) \rightarrow F(k)$ est bijective.

Soient $\mathscr{Z}, \mathscr{Z}^{\prime}$ deux c.c.o. de $\mathscr{X}_{R}$ sur $R$ telles que $\mathscr{Z}_{k}=\mathscr{Z}_{k}^{\prime}$. Il suffit de montrer que $\mathscr{Z}=\mathscr{Z}^{\prime}$ après une extension étale surjective de $R$. Comme $\mathscr{X} \rightarrow S$ est à fibres géométriques réduites, son lieu de lissité est un ouvert $S$-schématiquement dense. Par [EGA] IV.17.16.3, il existe une extension finie étale locale $\left(R^{\prime}, m^{\prime}, k^{\prime}\right)$ de $R$ et une section $g: \operatorname{Spec}\left(R^{\prime}\right) \rightarrow \mathscr{X}_{R^{\prime}}$ qui envoie le point fermé dans $\mathscr{Z}_{k}=\mathscr{Z}_{k}^{\prime}$. Il est alors clair que $\mathscr{Z}$ et $\mathscr{Z}^{\prime}$ sont égales à $\mathscr{C}(g)$, la c.c.o. de $\mathscr{X}_{R^{\prime}}$ le long de $g$.

Enfin, soit $\mathscr{Z}$ une c.c.o. de $\mathscr{X}_{k}$ sur $k$. On considère de nouveau une extension finie étale locale $\left(R^{\prime}, m^{\prime}, k^{\prime}\right)$ de $R$ et une section $g: \operatorname{Spec}\left(R^{\prime}\right) \rightarrow \mathscr{X}_{R^{\prime}}$ qui envoie le point fermé dans $\mathscr{Z}$. Il suit alors du lemme 2.2.1 que la composante connexe $\mathscr{C}(g)$ le long de $g$ est une c.c.o. de $\mathscr{X}_{R^{\prime}}$ qui relève $\mathscr{Z}_{k^{\prime}}$. D'après le lemme 2.3.1, son image dans $\mathscr{X}_{R}$ est une c.c.o. de $\mathscr{X}_{R}$ qui relève $\mathscr{Z}$, donc $\varphi$ est surjective.

(ii) Étant donné que pour tout point géométrique $\operatorname{Spec}(\Omega) \rightarrow S$ les composantes connexes de $\mathscr{X}_{\Omega} \times \mathscr{X}_{\Omega}$ sont les produits $\mathscr{C}_{i} \times \mathscr{C}_{j}$ des composantes connexes de $\mathscr{X}_{\Omega}$, il est immédiat que la relation $\mathscr{R}$ est représentable par la c.c.o. de $\mathscr{X} \times \mathscr{X}$ le long de la section diagonale. Par ailleurs, en associant à une section de $\mathscr{X}$ au-dessus d'un schéma $T$ la c.c.o. de $\mathscr{X} \times{ }_{S} T$ le long de cette section, on définit un morphisme surjectif $a: \mathscr{X} \rightarrow \pi_{0}(\mathscr{X} / S)$. Comme $\mathscr{X} \rightarrow S$ est plat et $\pi_{0}(\mathscr{X} / S) \rightarrow S$ étale, le morphisme $a$ est plat et donc un épimorphisme fppf. Il est clair que $a$ passe au quotient en un monomorphisme $\mathscr{X} / \mathscr{R} \rightarrow \pi_{0}(\mathscr{X} / S)$ qui est donc une immersion ouverte puis un isomorphisme.

(iii) Une fois qu'on a remarqué que le lieu unicomposante de $\mathscr{X} \times \mathscr{X}$ est $\mathscr{U} \times \mathscr{U}$, la preuve est formellement la même que celle du point (ii) en utilisant la proposition 2.2.4 au lieu de la proposition 2.2.1,

2.5.3 Remarques. (1) Il est facile de voir que $\mathscr{X}$ est un $S$-espace algébrique étale et quasi-compact si et seulement si $\mathscr{X} \rightarrow \pi_{0}(\mathscr{X} / S)$ est un isomorphisme. En particulier, on peut obtenir ainsi un exemple dans lequel $\pi_{0}(\mathscr{X} / S)$ n'est pas un schéma.

(2) Si $\pi_{0}(\mathscr{X} / S)$ est séparé, alors c'est un schéma par [Kn], chap. II, cor. 6.17. Mais ce foncteur est rarement séparé. Par exemple, dans les conditions du théorème, si $S$ est un trait strictement hensélien et $\mathscr{X}$ est connexe, il est facile de voir que $\pi_{0}(\mathscr{X} / S)$ est le schéma obtenu en recollant $n$ copies de $S$ le long de leur point ouvert commun, où $n$ est le nombre de composantes connexes de la fibre spéciale de $\mathscr{X}$. Ainsi $\pi_{0}(\mathscr{X} / S)$ n'est pas séparé si $n \geq 2$. 
(3) Il y a une description birationnelle de $\operatorname{Irr}(\mathscr{X} / S)$ qui mérite d'être mentionnée. Soit le foncteur $F$ dont les valeurs, pour chaque $S$-schéma $T$, sont les classes d'équivalence de sous-champs ouverts $\mathscr{U} \subset \mathscr{X}_{T}$, fidèlement plats et de présentation finie sur $T$, à fibres géométriquement irréductibles. Deux tels ouverts $\mathscr{U}, \mathscr{V}$ sont équivalents si et seulement si $\mathscr{U} \cap \mathscr{V}$ est surjectif sur $T$. Lorsque $\mathscr{X}$ est plat, de présentation finie, à fibres géométriquement réduites, il est clair que $F$ est isomorphe à $\operatorname{Irr}(\mathscr{X} / S)$; à l'aide de la proposition 2.2.4, on définit deux morphismes en sens opposés, inverses l'un de l'autre.

\subsection{Fonctorialité}

2.6.1 Corollaire. Soit $\mathscr{X}$ un $S$-champ algébrique plat, de présentation finie, à fibres géométriquement réduites. Alors il existe un morphisme surjectif $\operatorname{Irr}(\mathscr{X} / S) \rightarrow \pi_{0}(\mathscr{X} / S)$.

Preuve : Il est clair que le morphisme composé $\mathscr{U} \hookrightarrow \mathscr{X} \rightarrow \pi_{0}(\mathscr{X} / S)$ passe au quotient par la relation d'équivalence $\mathscr{S}$.

Nous appellerons application $S$-rationnelle de $\mathscr{X}$ vers $\mathscr{Y}$ une classe d'équivalence de morphismes $\mathscr{U} \rightarrow \mathscr{Y}$ définis sur des ouverts $S$-denses de $\mathscr{X}$, où l'on convient que deux morphismes $\mathscr{U} \rightarrow \mathscr{Y}$ et $\mathscr{V} \rightarrow \mathscr{Y}$ sont équivalents s'ils coïncident sur $\mathscr{U} \cap \mathscr{V}$. On trouve dans [EGA] IV.20 la terminologie de pseudo-morphisme de $\mathscr{X}$ dans $\mathscr{Y}$ relativement à $S$.

2.6.2 Corollaire. Soit $f: \mathscr{X} \rightarrow \mathscr{Y}$ une application $S$-rationnelle entre $S$-champs algébriques plats, de présentation finie, à fibres géométriquement réduites.

(i) $f$ induit un morphisme $\pi_{0}(f): \pi_{0}(\mathscr{X} / S) \rightarrow \pi_{0}(\mathscr{Y} / S)$.

(ii) $S i f^{-1}\left(\mathscr{U}_{\mathscr{Y}}\right) \subset \mathscr{U}_{\mathscr{X}}, f$ induit un morphisme $\operatorname{Irr}(f): \operatorname{Irr}(\mathscr{X} / S) \rightarrow \operatorname{Irr}(\mathscr{Y} / S)$.

On notera que, compte tenu du caractère birationnel de $\operatorname{Irr}(\mathscr{X} / S)$, l'assertion de (ii) est encore valable sous l'hypothèse plus faible que $f^{-1}\left(\mathscr{U}_{\mathscr{Y}}\right) \cap \mathscr{U}_{\mathscr{X}}$ est $S$-dense dans $\mathscr{U}_{\mathscr{X}}$, par restriction à cet ouvert.

Preuve : Pour un champ $\mathscr{X}$, notons $\mathscr{U}_{\mathscr{X}}, \mathscr{R}_{\mathscr{X}}, \mathscr{S}_{\mathscr{X}}$ les objets définis dans le théorème 2.5.2. Supposons d'abord que $f$ est partout définie, i.e. un morphisme $f: \mathscr{X} \rightarrow \mathscr{Y}$. Dans ce cas, il est clair que le morphisme composé $\mathscr{X} \rightarrow \mathscr{Y} \rightarrow \pi_{0}(\mathscr{Y} / S)$ passe au quotient par la relation d'équivalence $\mathscr{R}_{\mathscr{X}}$ en un morphisme $\pi_{0}(\mathscr{X} / S) \rightarrow \pi_{0}(\mathscr{Y} / S)$. De même, si $f^{-1}\left(\mathscr{U}_{\mathscr{Y}}\right) \subset \mathscr{U}_{\mathscr{X}}$ alors le morphisme composé $\mathscr{U}_{\mathscr{X}} \rightarrow \mathscr{U}_{\mathscr{Y}} \rightarrow \operatorname{Irr}(\mathscr{Y} / S)$ passe au quotient par la relation d'équivalence $\mathscr{S}_{\mathscr{X}}$ en un morphisme $\operatorname{Irr}(\mathscr{X} / S) \rightarrow \operatorname{Irr}(\mathscr{Y} / S)$.

Si $f$ est une immersion ouverte $S$-dense, les morphismes ci-dessus sont étales et bijectifs sur les fibres géométriques, donc ce sont des isomorphismes. On en déduit que tout ce qui vient d'être dit est encore valable pour une application $S$-rationnelle $f: \mathscr{X} \rightarrow \mathscr{Y}$ quelconque, ce qui termine de démontrer (i) et (ii).

2.6.3 Corollaire. Soit $\mathscr{X}$ un $S$-champ algébrique plat, de présentation finie, à fibres géométriquement réduites. On suppose que $\mathscr{X}$ est un champ algébrique modéré au sens de [AOV] et on note $p: \mathscr{X} \rightarrow X$ son espace grossier de modules. Alors $X$ est un $S$-espace algébrique plat, de présentation finie, à fibres géométriquement réduites et l'application induite $\pi_{0}(\mathscr{X} / S) \rightarrow \pi_{0}(X / S)$ est un isomorphisme. 
Preuve : D'après [AOV], corollary 3.3, l'espace $X$ est plat sur $S$ et ses fibres géométriques sont les espaces de modules des fibres géométriques de $\mathscr{X}$. Ceci montre que $X$ est à fibres géométriquement réduites. L'application induite par fonctorialité de $p: \mathscr{X} \rightarrow X$ est clairement bijective sur les fibres géométriques, donc c'est un isomorphisme.

\section{Composantes fermées}

\subsection{Définitions}

Pour un champ $\mathscr{X} \rightarrow S$ à fibres non (géométriquement) réduites, le foncteur des c.c.o. n'est pas nécessairement représentable. Par exemple, considérons un anneau de valuation discrète complet à corps résiduel algébriquement clos $(R, K, k, \pi)$ et prenons pour $\mathscr{X}$ la réunion de deux sections de la droite affine sur $R$ qui se rencontrent dans la fibre spéciale, par exemple le spectre de $R[x] /\left(x^{2}-\pi^{n} x\right)$. Il est clair que $\pi_{0}(\mathscr{X} / S)(R)=\emptyset$ de sorte que l'approximation des composantes connexes formelles est prise en défaut. Il en découle que $\pi_{0}(\mathscr{X} / S)$ n'est pas représentable par un espace algébrique. Néanmoins, dans cet exemple le champ $\mathscr{X}$ est propre. Grâce à l'existence du schéma de Hilbert, pour ces champs, on a des notions utiles de composantes connexes et irréductibles fermées, ainsi que nous le voyons maintenant.

3.1.1 Définitions. Soit $\mathscr{X}$ un champ algébrique de présentation finie sur un schéma $S$.

(1) Une composante connexe (relative) fermée (en abrégé c.c.f.) de $\mathscr{X}$ sur $S$ est un sous-champ $\mathscr{C} \subset \mathscr{X}$ fermé, plat et de présentation finie sur $S$, tel que le support de $\mathscr{C}_{s}$ est une composante connexe de $\mathscr{X}_{s}$, pour tout point géométrique $s$ de $S$. On note $\pi_{0}(\mathscr{X} / S)^{\mathfrak{f}}$ le foncteur des c.c.f. de $\mathscr{X}$ sur $S$.

(2) On dit qu'une c.c.f. $\mathscr{C}$ est réduite si ses fibres géométriques sont réduites. On note $\pi_{0}(\mathscr{X} / S)^{\mathfrak{r}}$ le sous-foncteur correspondant de $\pi_{0}(\mathscr{X} / S)^{\mathfrak{f}}$.

(3) Une composante irréductible (relative) fermée (en abrégé c.i.f.) de $\mathscr{X}$ sur $S$ est un sous-champ $\mathscr{I} \subset \mathscr{X}$ fermé, plat et de présentation finie sur $S$, tel que le support de $\mathscr{I}_{s}$ est une composante irréductible de $\mathscr{X}_{s}$, pour tout point géométrique $s$ de $S$. On note $\operatorname{Irr}(\mathscr{X} / S)^{\mathfrak{f}}$ le foncteur des c.i.f. de $\mathscr{X}$ sur $S$.

Lorsque $\mathscr{X} / S$ est à fibres géométriquement réduites, ces foncteurs se comparent aux foncteurs de composantes ouvertes (voir proposition 3.2.4). Dans le cas contraire, on s'attend bien sûr à ce que les foncteurs de composantes fermées soient ramifiés. L'exemple 3.1.2(1) ci-dessous montre que $\pi_{0}(\mathscr{X} / S)^{\mathfrak{f}}$ peut être démesurément gros, de dimension positive et non quasi-compact, même si $\mathscr{X} / S$ est propre. Ceci est dû à la présence de composantes immergées dans les c.c.f. C'est ce qui justifie l'introduction de $\pi_{0}(\mathscr{X} / S)^{\mathfrak{r}}$ qui sera, lui, quasi-fini. Pour les composantes irréductibles, la situation est différente car, comme on va le voir, en présence de fibres non réduites, on ne sait pas en fabriquer de bons espaces de modules.

3.1.2 Exemples. (1) Soient $k$ un corps et $X_{0}$ un $k$-schéma géométriquement connexe. Soit $X=$ $X_{0}[\epsilon]$ obtenu à partir de $X$ par le changement de base de $k$ à l'anneau des nombres duaux $k[\epsilon] /\left(\epsilon^{2}\right)$. On a alors un isomorphisme de foncteurs $\operatorname{Hilb}\left(X_{0} / k\right) \simeq \pi_{0}(X / k)^{\mathfrak{f}}$ qui envoie le sous-schéma fermé d'idéal J sur la c.c.f. d'idéal $\epsilon \mathcal{J}$.

(2) Si $\mathscr{X} / S$ n'est pas séparé, les éléments formels des foncteurs $\pi_{0}(\mathscr{X} / S)^{\mathfrak{f}}$ et $\operatorname{Irr}(\mathscr{X} / S)^{\mathfrak{f}}$ ne peuvent pas être approximés en général, même avec $\mathscr{X} / S$ plat et pur. Par exemple, prenons pour base un 
anneau de valuation discrète $(R, K, k, \pi)$ complet à corps résiduel algébriquement clos et prenons pour $X$ le schéma obtenu en recollant deux copies $U, V$ de la droite affine sur $R$ le long de leurs fibres génériques. Pour tout $n \geq 1$, posons $R_{n}=R / \pi^{n}, X_{n}=X \otimes R_{n}, U_{n}=U \otimes R_{n}, V_{n}=V \otimes R_{n}$. Alors les ouverts $U_{n}$ et $V_{n}$ sont disjoints dans $X_{n}$, et la collection $\left(U_{n}\right)$ définit une c.c.f. (ou une c.i.f.) élément de $\lim _{0} \pi_{0}(X / R)^{\mathfrak{f}}\left(R_{n}\right)$. Or on voit que $\pi_{0}(X / R)^{\mathfrak{f}}$ n'a pas de sections sur $R$, si bien que la composante formelle $\left(U_{n}\right)$ ne peut pas être approximée. Les foncteurs $\pi_{0}(\mathscr{X} / S)^{\mathfrak{f}}$ et $\operatorname{Irr}(\mathscr{X} / S)^{\mathfrak{f}}$ ne sont donc pas représentables.

(3) Si $\mathscr{X}$ est séparé non propre, les espaces tangents et des espaces d'obstructions de la théorie de déformations de $\pi_{0}(\mathscr{X} / S)^{\mathfrak{f}}$ ne sont pas de dimension finie en général. Par exemple, soit $X=X_{0}[\epsilon]$ comme dans l'exemple (1) et soit $Y=X_{0} \in \pi_{0}(\mathscr{X} / S)^{\mathfrak{f}}(k)$. Alors, l'espace tangent au foncteur de déformations de $Y$ est $\operatorname{Hom}_{\mathcal{O}_{X}}\left(\mathcal{J}_{Y}, \mathcal{O}_{X} / \mathcal{J}_{Y}\right) \simeq H^{0}\left(X_{0}, \mathcal{O}_{X_{0}}\right)$ et l'espace d'obstructions est $\operatorname{Ext}_{\mathcal{O}_{X}}^{1}\left(\mathcal{J}_{Y}, \mathcal{O}_{X} / \mathcal{J}_{Y}\right) \simeq \operatorname{Ext}_{\mathcal{O}_{X_{0}}}^{1}\left(\mathcal{O}_{X_{0}}, \mathcal{O}_{X_{0}}\right)$ qui ne sont pas de dimension finie en général.

3.1.3 Lemme. Pour tout $S$-champ algébrique de présentation finie $\mathscr{X}$, le foncteur $\pi_{0}(\mathscr{X} / S)^{\mathfrak{r}}$ est localement de présentation finie, quasi-compact et à fibres finies.

Preuve : Le fait que $\pi_{0}(\mathscr{X} / S)^{\mathfrak{r}}$ est localement de présentation finie provient des résultats habituels de [EGA] IV.8.6 et de leur adaptation aux champs. Pour montrer qu'il est quasi-compact et à fibres finies, on peut faire quelques réductions : on peut supposer que $S$ est affine quitte à localiser, qu'il est de type fini sur $\mathbb{Z}$ puisque $\mathscr{X}$ provient par changement de base d'un tel schéma, et enfin que $S$ est réduit et irréductible, quitte à remplacer $S$ par une de ses composantes irréductibles réduites. Notons $\eta$ le point générique de $S$. Par récurrence noethérienne, il suffit de trouver un voisinage de $\eta$ au-dessus duquel $\pi_{0}(\mathscr{X} / S)^{\mathfrak{r}}$ est quasi-compact. On procède alors exactement comme dans la démonstration du lemme 2.1.3. Les seules modifications sont le choix de l'extension finie $K / k(\eta)$ qui doit être telle que les sous-schémas fermés réduits des composantes connexes de $\mathscr{X}_{\eta} \otimes K$ soient géométriquement connexes et géométriquement réduits. Ensuite, on utilise A.2.2 (iv) et A.2.6 (ii)(iii) pour trouver un voisinage de $\eta$ au-dessus duquel on dipose de $r$ composantes connexes réduites relatives $\mathscr{C}_{i}$ pour $\mathscr{X}$. On trouve un morphisme surjectif $S \times\{1, \ldots, r\} \rightarrow \pi_{0}(\mathscr{X} / S)^{\mathfrak{r}}$ ce qui prouve notre assertion. Noter que contrairement à ce qu'il se passe dans le lemme 2.1.3, on ne peut pas prouver que ce morphisme est étale ou même plat.

\subsection{Représentabilité}

3.2.1 Théorème. Soit $\mathscr{X}$ un $S$-champ algébrique propre et de présentation finie.

(1) Le foncteur $\pi_{0}(\mathscr{X} / S)^{\mathfrak{f}}$ est représentable par un $S$-espace algébrique formel localement de présentation finie et séparé.

(2) Le foncteur $\pi_{0}(\mathscr{X} / S)^{\mathfrak{r}}$ est représentable par un $S$-schéma formel quasi-fini et séparé.

Preuve : Les deux foncteurs sont localement de présentation finie, par les arguments habituels. Il est clair, d'après le critère valuatif, qu'ils sont séparés sur $S$ : en effet, si $R$ est un anneau de valuation discrète de corps de fractions $K$, une c.c.f. $\mathscr{C}_{R}$ de $\mathscr{X} \times{ }_{S} \operatorname{Spec}(R)$ est uniquement déterminée comme l'adhérence schématique dans $\mathscr{X}$ de sa fibre générique $\mathscr{C}_{K}$.

Montrons que $\pi_{0}(\mathscr{X} / S)^{\mathfrak{f}}$ est représentable par un $S$-espace algébrique formel. Soit $H_{\mathscr{X} / S}$ le foncteur de Hilbert des sous-champs fermés de $\mathscr{X}$ sur $S$ : c'est le foncteur Quot associé au faisceau $\mathcal{O}_{\mathscr{X}}$, représentable par un espace algébrique séparé, localement de présentation finie et vérifiant le critère valuatif de propreté (voir [Ol], th. 1.5). Par ailleurs, pour un sous-champ fermé $\mathscr{W} \subset \mathscr{X}$ de complémentaire ouvert $\mathscr{U}$, il est équivalent de dire que les fibres $\mathscr{W}_{s}$ ont un support ouvert dans $\mathscr{X}_{s}$ 
ou que les fibres de $\mathscr{U}$ ont un support propre. D'après le lemme 3.2 .2 ci-dessous, cette condition est représentée par un sous-champ ouvert $K$ de $H_{\mathscr{X} / S}$. Ensuite, on note que le lieu des points de $S$ tels que la fibre $\mathscr{W}_{s}$ est géométriquement connexe est un fermé de $K$ (voir [EGA], IV.15.5.9); notons $K_{0}$ le sous-espace fermé réduit que ce fermé détermine. Un morphisme $f: T \rightarrow K$ définit une c.c.f. de $\mathscr{X}_{T}$ sur $T$ si et seulement si son image ensembliste tombe dans $K_{0}$. Il est équivalent de dire que l'image schématique $Z$ de $f$ vérifie $Z_{\text {red }} \subset K_{0}$, ou encore que l'idéal $\mathcal{J}$ de $K_{0}$ dans $K$ est inclus dans la racine de l'idéal $\mathcal{J}$ de $Z$. Or $K$ est somme disjointe d'espaces algébriques de type fini $K^{i}$, de sorte que pour chaque $i$ on obtient l'existence d'un entier $n_{i}$ tel que $\left(\mathcal{J}_{\mid K_{i}}\right)^{n_{i}} \subset \mathcal{J}_{\mid K_{i}}$, c'est-à-dire qu'en restriction à $K_{i}$, l'image de $f$ est dans le $n_{i}$-ième voisinage de $K_{0} \cap K^{i}$ dans $K^{i}$. Finalement $\pi_{0}(\mathscr{X} / S)^{\mathfrak{f}}$ est représentable par la somme des complétés de $K^{i}$ le long de $K_{0} \cap K^{i}$.

Concernant le foncteur $\pi_{0}(\mathscr{X} / S)^{\mathfrak{r}}$, on note d'abord que la composante connexe universelle $\mathscr{C}^{u} \rightarrow \pi_{0}(\mathscr{X} / S)^{\mathfrak{r}}$ est un morphisme propre donc pur. On peut lui appliquer le théorème B.4(i) et conclure que le foncteur $\pi_{0}(\mathscr{X} / S)^{\mathfrak{r}}$ est représentable par le sous-espace ouvert de $\pi_{0}(\mathscr{X} / S)^{\mathfrak{f}}$ au-dessus duquel $\mathscr{C}^{u}$ est à fibres géométriquement réduites. De plus $\pi_{0}(\mathscr{X} / S)^{\mathfrak{r}}$ est quasi-compact d'après le lemme 3.1.3, donc il est quasi-fini. Il s'ensuit que cet espace algébrique formel est en fait un schéma formel : étant donné que ses tronqués sont quasi-finis et séparés sur $S$, ceci provient en effet de $[\mathrm{Kn}]$, chap. II, cor. 6.17.

Dans la preuve, on a utilisé le lemme suivant :

3.2.2 Lemme. Soit $\mathscr{X}$ un $S$-champ algébrique propre, de présentation finie, et $\mathscr{U}$ un ouvert de $\mathscr{X}$ de présentation finie sur $S$. Alors, le lieu $S_{0}$ des points $s \in S$ tels que $\mathscr{U}_{s}$ est propre sur $k(s)$ est un ouvert, et la restriction $\mathscr{U} \times_{S} S_{0} \rightarrow S_{0}$ est propre.

Preuve : Par les arguments standard, on peut supposer $S$ noethérien. En suivant la lecture du début du paragraphe 15.7 de [EGA] IV, on voit que les résultats des numéros 15.7.1 à 15.7.7 sont valables avec pour seule modification de remplacer le critère valuatif de propreté pour les schémas par son analogue pour les champs ([LMB] chapitre 7 et [01], introduction).

On peut donc se ramener au cas où $S$ est le spectre d'un anneau de valuation discrète à corps résiduel algébriquement clos (voir en particulier [EGA] IV.15.7.5 et 15.7.7). Il suffit de montrer que si la fibre $\mathscr{U}_{s}$ au-dessus du point fermé $s \in S$ est propre sur $k(s)$ (on la suppose non vide, sans quoi il n'y a rien à démontrer), alors $\mathscr{U}$ est propre sur $S$. Puisque $S$ est strictement hensélien, le schéma $S^{\prime}$ de la factorisation de Stein $\mathscr{X} \rightarrow S^{\prime}=\operatorname{St}(\mathscr{X} / S) \rightarrow S$ est somme disjointe d'un nombre fini de $S$-schémas locaux finis $S_{1}^{\prime}, \ldots, S_{n}^{\prime}$. On peut remplacer $S$ par l'un des $S_{i}^{\prime}$, puis par une composante irréductible réduite $T$ de $S_{i}^{\prime}$ ([EGA] II.5.4.5) surjective sur $S$ (sans quoi il n'y a rien à démontrer) et enfin par la normalisation $\widetilde{T}$ puisque celle-ci est finie sur $T$. On s'est donc ramené au cas où $S$ est le spectre d'un anneau de valuation discrète et $\mathscr{X} \rightarrow S$ est à fibres géométriquement connexes.

Par hypothèse $\mathscr{U}_{s}$ est propre sur $k(s)$, donc ouvert et fermé non vide dans $\mathscr{X}_{s}$, donc $\mathscr{U}_{s}=\mathscr{X}_{s}$. Par suite, le complémentaire $\mathscr{Z}=\mathscr{X} \backslash \mathscr{U}$ est inclus dans la fibre ouverte de $\mathscr{X}$. Comme $\mathscr{Z}$ est propre sur $S$, ceci n'est possible que si $\mathscr{Z}=\emptyset$, ce qui montre que $\mathscr{U}=\mathscr{X}$.

3.2.3 Remarque. Contrairement au cas du foncteur des composantes ouvertes avec $\mathscr{X}$ à fibres géométriquement réduites, ici il n'existe pas en général de morphisme $\mathscr{X} \rightarrow \pi_{0}(\mathscr{X} / S)^{\mathfrak{f}}$ ou $\mathscr{X} \rightarrow$ $\pi_{0}(\mathscr{X} / S)^{\mathfrak{r}}$. Par exemple, soit $S$ le spectre d'un corps imparfait $k$ de caractéristique $p>0$, soit $t \notin k^{p}$ et $C$ la courbe de type Fermat d'équation $x^{p}+y^{p}=t z^{p}$. On peut voir que $\pi_{0}(C / k)^{\mathfrak{r}} \simeq \operatorname{Spec}(k(\sqrt[p]{t}))$ et il n'y a pas de morphisme $C \rightarrow \pi_{0}(C / k)^{\mathfrak{r}}$.

3.2.4 Proposition. Soit $\mathscr{X}$ un $S$-champ algébrique de présentation finie, plat, à fibres géométriquement réduites. 
(1) On a $\pi_{0}(\mathscr{X} / S)^{\mathfrak{f}}=\pi_{0}(\mathscr{X} / S)^{\mathfrak{r}} \subset \pi_{0}(\mathscr{X} / S)$.

(2) On a un monomorphisme de foncteurs $\operatorname{Irr}(\mathscr{X} / S)^{\mathfrak{f}} \hookrightarrow \operatorname{Irr}(\mathscr{X} / S)$.

(3) Si de plus $\mathscr{X}$ est plat et pur sur $S$, les monomorphismes

$$
\pi_{0}(\mathscr{X} / S)^{\mathfrak{f}} \subset \pi_{0}(\mathscr{X} / S) \quad \text { et } \quad \operatorname{Irr}(\mathscr{X} / S)^{\mathfrak{f}} \hookrightarrow \operatorname{Irr}(\mathscr{X} / S)
$$

sont des immersions ouvertes.

Preuve : (1) Le fait que $\pi_{0}(\mathscr{X} / S)^{\mathfrak{f}}=\pi_{0}(\mathscr{X} / S)^{\mathfrak{r}}$ est clair. Pour montrer que $\pi_{0}(\mathscr{X} / S)^{\mathfrak{f}} \subset \pi_{0}(\mathscr{X} / S)$, il suffit d'observer que toute c.c.f. $\mathscr{C} \subset \mathscr{X}$ est ouverte dans $\mathscr{X}$. On peut vérifier ceci localement sur $S$, or comme le lieu lisse de $\mathscr{C}$ est $S$-dense, on peut supposer qu'on dispose d'une section $g: S \rightarrow \mathscr{C}$. Dans ce cas $\mathscr{C}$ est égale à la composante connexe de $\mathscr{X}$ le long de la section $g$, qui est ouverte d'après la proposition 2.2.1.

(2) Soit $\mathscr{U}$ le sous-champ ouvert de $\mathscr{X}$ égal au lieu unicomposante. Si $\mathscr{I}$ est une c.i.f. de $\mathscr{X} / S$, le sous-champ $\mathscr{I} \cap \mathscr{U}$ est ouvert dans $\mathscr{X}$. En effet, en procédant comme dans (1) on voit qu'après choix d'une section locale $g: S \rightarrow \mathscr{I} \cap \mathscr{U}$, ce sous-champ est égal à la composante irréductible ouverte de $\mathscr{X}$ le long de la section $g$, qui est ouverte (proposition 2.2.4). Le morphisme $\operatorname{Irr}(\mathscr{X} / S)^{\mathfrak{f}} \hookrightarrow \operatorname{Irr}(\mathscr{X} / S)$ est défini par $\mathscr{I} \mapsto \mathscr{I} \cap \mathscr{U}$. Comme de plus $\mathscr{I} \cap \mathscr{U}$ est plat sur $S$ et dense dans $\mathscr{I}$ fibre à fibre, il est schématiquement dense dans $\mathscr{I}$. Ainsi $\mathscr{I}$ est égal à l'adhérence schématique de $\mathscr{I} \cap \mathscr{U}$ dans $\mathscr{X}$. Ceci montre que le morphisme est un monomorphisme.

(3) Soit $\mathscr{C}^{u}$ la c.c.o. universelle au-dessus de $\pi_{0}(\mathscr{X} / S)$. D'après ce qui précède $\pi_{0}(\mathscr{X} / S)^{\mathfrak{f}}$ est représentable par le sous-espace de $\pi_{0}(\mathscr{X} / S)$ des points où l'adhérence schématique $\overline{\mathscr{C}}^{u}$ de $\mathscr{C}^{u}$ dans $\mathscr{X} \times \pi_{0}(\mathscr{X} / S)$ est plate sur $S$ et à fibres géométriquement connexes. Or, le lieu de platitude de $\overline{\mathscr{C}}^{u} \rightarrow S$ est un ouvert de $S$. Restreignons-nous à cet ouvert et supposons donc $\overline{\mathscr{C}}^{u}$ plat sur $S$. On vérifie immédiatement que $\overline{\mathscr{C}}^{u}$ est pur sur $S$ : en effet, la formation de l'adhérence schématique commute au changement de base plat de sorte qu'on peut supposer que $S$ est local hensélien; comme $\mathscr{X}$ est pur sur $S$, pour tout point $x$ de $\overline{\mathscr{C}}^{u}$ qui est associé dans sa fibre, l'adhérence de $x$ (qui est incluse dans $\overline{\mathscr{C}}^{u}$ car celui-ci est fermé) rencontre la fibre fermée de $\mathscr{X}$ en un point qui appartient donc à la fibre fermée de $\overline{\mathscr{C}}^{u}$, comme souhaité. Alors, le lieu des points où la fibre est géométriquement connexe est ouvert d'après le thérorème B.4(ii), ce qui conclut. On procède pareil pour montrer que $\operatorname{Irr}(\mathscr{X} / S)^{\mathfrak{f}} \hookrightarrow \operatorname{Irr}(\mathscr{X} / S)$ est une immersion ouverte.

3.2.5 Proposition. Soit $\mathscr{X}$ un $S$-champ algébrique propre, plat, de présentation finie et à fibres géométriquement réduites.

(1) Soit $\mathscr{X} \rightarrow \operatorname{St}(\mathscr{X} / S) \rightarrow S$ la factorisation de Stein. Alors, on a des isomorphismes

$$
\operatorname{St}(\mathscr{X} / S) \simeq \pi_{0}(\mathscr{X} / S)^{\mathfrak{f}}=\pi_{0}(\mathscr{X} / S)^{\mathfrak{r}}=\pi_{0}(\mathscr{X} / S) .
$$

(2) L'ouvert $\operatorname{Irr}(\mathscr{X} / S)^{\mathfrak{f}} \subset \operatorname{Irr}(\mathscr{X} / S)$ (voir 3.2.4(3)) est un schéma étale et séparé.

Preuve : (1) Notons qu'un champ propre est pur. Le lemme 3.2 .2 montre qu'une c.c.o. $\mathscr{C} \in$ $\pi_{0}(\mathscr{X} / S)$, qui est propre fibre à fibre, est propre sur $S$. Ainsi, l'inclusion $\pi_{0}(\mathscr{X} / S)^{\mathfrak{f}} \subset \pi_{0}(\mathscr{X} / S)$ de $3.2 .4(3)$ est en fait une égalité. Notons maintenant $S^{\prime}:=\operatorname{St}(\mathscr{X} / S)$. Pour tout point $s \in S$, l'algèbre $H^{0}\left(\mathscr{X}_{s}, \mathcal{O}_{\mathscr{X}_{s}}\right)$ est étale car la fibre $\mathscr{X}_{s}$ est géométriquement réduite. Il en découle que $f: \mathscr{X} \rightarrow S$ est cohomologiquement plat en dimension 0 et que $f_{*} \mathcal{O}_{\mathscr{X}}$ est une $\mathcal{O}_{S^{-} \text {-algèbre étale }}$ ([EGA] III.7.8.6 et 7.8.7). Ainsi $S^{\prime} \rightarrow S$ est étale, donc le morphisme $\mathscr{X} \rightarrow S^{\prime}$ est plat. Soit $s^{\prime}$ un 
point géométrique de $S^{\prime}$ et $s$ son image dans $S$. D'après les propriétés de la factorisation de Stein, les fibres de $\mathscr{X} \rightarrow S^{\prime}$ sont des composantes connexes de $\mathscr{X}_{s}$ de sorte que finalement $\mathscr{X}$ est une c.c.f. de $\mathscr{X} \times{ }_{S} S^{\prime} / S^{\prime}$ correspondant à un morphisme $S^{\prime} \rightarrow \pi_{0}(\mathscr{X} / S)^{\mathfrak{f}}$. Ce morphisme est un morphisme entre deux schémas finis étales, qui est un isomorphisme fibre à fibre (noter que la formation de $\operatorname{St}(\mathscr{X} / S)$ commute au changement de base), donc c'est un isomorphisme.

(2) Le fait que $\operatorname{Irr}(\mathscr{X} / S)^{\mathfrak{f}}$ est séparé provient du fait que sur un anneau de valuation discrète de base, une c.i.f. est uniquement déterminée comme adhérence schématique dans $\mathscr{X}$ de sa fibre générique. Comme $\operatorname{Irr}(\mathscr{X} / S)^{\mathfrak{f}}$ est étale et séparé, c'est alors un schéma.

La fonctorialité en $\mathscr{X}$ pour $\pi_{0}(\mathscr{X} / S)^{\mathfrak{f}}$ est évidemment moins bonne que pour $\pi_{0}(\mathscr{X} / S)$. Nous terminons cette section avec deux cas simples de covariance et de contravariance :

3.2.6 Proposition. Soient $\mathscr{X}$ et $\mathscr{Y}$ des $S$-champs algébriques plats et de présentation finie.

(1) Tout morphisme fini étale $f: \mathscr{X} \rightarrow \mathscr{Y}$ induit un morphisme $f_{*}: \pi_{0}(\mathscr{X} / S)^{\mathfrak{f}} \rightarrow \pi_{0}(\mathscr{Y} / S)^{\mathfrak{f}}$.

(2) Tout morphisme fppf à fibres géométriquement connexes $f: \mathscr{X} \rightarrow \mathscr{Y}$ induit un morphisme $f^{*}: \pi_{0}(\mathscr{Y} / S)^{\mathfrak{f}} \rightarrow \pi_{0}(\mathscr{X} / S)^{\mathfrak{f}}$.

Preuve : Soit $f: \mathscr{X} \rightarrow \mathscr{Y}$ un morphisme avec $\mathscr{X}, \mathscr{Y}$ plats et de présentation finie.

(1) Le morphisme covariant $f_{*}$ envoie une c.c.f. $\mathscr{C}$ sur l'image schématique $\mathscr{D}:=f(\mathscr{C})$. Comme $f$

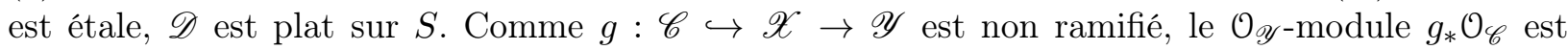
localement monogène, donc son annulateur est égal à $\operatorname{Fitt}_{0}\left(g_{*} \mathcal{O}_{\mathscr{C}}\right)$, le 0-ième idéal de Fitting ([Ei], prop. 20.7), et ceci reste vrai après tout changement de base. Comme la formation des idéaux de Fitting commute au changement de base, il en va de même pour la formation de $\mathscr{D}$. Enfin, comme $f$ est ouvert et fermé, le sous-champ $\mathscr{D} \subset \mathscr{Y}$ est à fibres géométriquement connexes, ouvertes et fermées donc finalement c'est bien une c.c.f. de $\mathscr{Y} / S$.

(2) Le morphisme contravariant $f^{*}$ envoie une c.c.f. $\mathscr{D} \subset \mathscr{Y}$ sur $\mathscr{C}:=f^{-1}(\mathscr{D})$. Il est clair que $\mathscr{C}$ est plat et de présentation finie sur $S$, et comme $f$ est universellement submersif à fibres géométriquement connexes, ses fibres géométriques qui sont ouvertes et fermées sont aussi connexes.

\subsection{Un contre-exemple}

Dans cette sous-section, nous montrons que pour un $S$-champ algébrique propre, plat et de présentation finie, le foncteur $\operatorname{Irr}(\mathscr{X} / S)^{\mathfrak{f}}$ n'est pas en général représentable par un $S$-espace algébrique formel. Le contre-exemple est donné dans 3.3.6. Il est basé sur la propriété 3.3.1 ci-dessous, qui est peut-être bien connue mais dont je n'ai trouvé mention nulle part dans la littérature. Notons que les critères d'Artin montrent assez facilement que le foncteur $\operatorname{Irr}(\mathscr{X} / S)^{\mathfrak{f}}$ de l'exemple 3.3.6 n'est pas représentable par un $S$-espace algébrique. Malheureusement, les critères d'Artin ne s'étendent pas aux espaces algébriques formels (notamment, l'axiome d'approximation des éléments formels fait défaut). C'est pourquoi nous avons recours à une idée différente.

Soit $P=P(\mathscr{X} / k)$ une propriété des champs algébriques $\mathscr{X}$ de type fini sur un corps $k$ qui est invariante par extension du corps de base, au sens où $\mathscr{X}$ vérifie $P$ si et seulement si $\mathscr{X} \otimes_{k} \ell$ vérifie $P$, pour toute extension de corps $\ell / k$. On dira aussi que $P$ est une propriété géométrique. À tout champ algébrique $\mathscr{X}$ de présentation finie sur un schéma $S$, on peut associer :

(1) l'ensemble $E_{P}=E_{P}(\mathscr{X} / S)$ des $s \in S$ tels que la fibre de $\mathscr{X}$ en $s$ vérifie $P$, et 
(2) le foncteur $F_{P}=F_{P}(\mathscr{X} / S)$ sur la catégorie des $S$-schémas défini par

$$
F_{P}(T)=\left\{\begin{array}{cl}
\{\emptyset\} & \text { si les fibres de } X \times_{S} T \rightarrow T \text { vérifient } P \\
\emptyset & \text { sinon. }
\end{array}\right.
$$

On appellera $E_{P}$ le lieu indicateur et $F_{P}$ le foncteur indicateur de la propriété $P$ pour $\mathscr{X} / S$.

3.3.1 Proposition. Avec les notations ci-dessus :

(1) $F_{P}$ est représentable par un schéma si et seulement si $E_{P}$ est ouvert dans $S$.

(2) $F_{P}$ est représentable par un schéma formel si et seulement si $E_{P}$ est localement fermé dans $S$.

3.3.2 Remarques. (1) Si $S$ est un espace algébrique au lieu d'un schéma, le même énoncé est valable (avec la même preuve) en remplaçant «schéma » par « espace algébrique » et « schéma formel » par « espace algébrique formel ».

(2) La preuve utilise trois petits lemmes (3.3.3, 3.3.4 et 3.3.5) qui seront établis ci-dessous.

Preuve : La formation de $E_{P}$ et de $F_{P}$ commute aux changements de base $S^{\prime} \rightarrow S$. Comme les termes des équivalences à démontrer sont des propositions de nature locale sur $S$, on peut supposer $S$ affine. Par ailleurs, comme $\mathscr{X}$ est de présentation finie sur $S$, il en va de même pour le foncteur $F_{P}$, et on peut donc supposer que $S$ est le spectre d'un anneau noethérien.

(1) Si $E_{P}$ est ouvert dans $S$, alors c'est un sous-schéma de $S$ et il est clair qu'il représente $F_{P}$. Réciproquement, si $F_{P}$ est représentable par un schéma, alors la partie $E_{P}$, qui est l'image de $F_{P} \rightarrow S$, est constructible. Pour montrer que $E_{P}$ est ouvert, il suffit de montrer qu'il est stable par générisation. Pour cela, on peut remplacer $S$ par le spectre d'un anneau de valuation discrète $(R, m)$, dont le point fermé est dans $E_{P}$, et il faut montrer que $E_{P}=S$. Or par hypothèse $F_{P}\left(R / m^{n}\right)=\{\emptyset\}$ pour tout $n \geq 1$. Comme aucune immersion fermée $f: X \rightarrow S$ distincte de l'identité ne se factorise à travers tous les voisinages infinitésimaux $\operatorname{Spec}\left(R / m^{n}\right)$, le lemme 3.3.3 ci-dessous montre que l'image de $F_{P} \rightarrow S$ est nécessairement égale à $S$, donc $E_{P}=S$.

(2) Si $E_{P}$ est localement fermé dans $S$, il est fermé dans un ouvert $U \subset S$. Pour montrer que $F_{P}$ est représentable par un schéma formel, on peut remplacer $S$ par $U$ et supposer que $E_{P}$ est fermé. On note encore $E_{P}$ le sous-schéma fermé réduit de $S$ de support $E_{P}$. Un morphisme $f: T \rightarrow S$ définit un point de $F_{P}$ si et seulement si son image ensembliste tombe dans $E_{P}$. Il est équivalent de dire que l'image schématique $Z$ de $f$ vérifie $Z_{\text {red }} \subset E_{P}$, ou encore que l'idéal J de $E_{P}$ dans $K$ est inclus dans la racine de l'idéal $\mathcal{J}$ de $Z$. Or $E_{P}$ est noethérien, donc il existe un entier $n$ tel que $\mathfrak{J}^{n} \subset \mathcal{J}$, ce qui signifie que $f$ se factorise par le $n$-ième voisinage de $E_{P}$ dans $S$. Finalement $F_{P}$ est représentable par le complété de $S$ le long de $E_{P}$.

Réciproquement, supposons que $F_{P}$ est représentable par un schéma formel. Il suffit de montrer que $E_{P}$ est ouvert dans son adhérence. On peut donc remplacer $S$ par l'adhérence réduite de $E_{P}$ dans $S$ et $X$ par sa restriction à cette adhérence. Alors $E_{P}$ est dense dans $S$ et on doit montrer qu'il est ouvert. Il suffit de montrer qu'il est stable par générisation. Soit $x_{2} \in E_{P}$ et $x_{1} \in S$ une générisation de $x$. Comme $E_{P}$ est dense dans $S$, il existe une générisation $x_{0}$ de $x_{1}$ qui appartient à $E_{P}$. D'après le lemme 3.3.4, il existe un schéma $\Sigma$, spectre d'un anneau de valuation de rang 2 , de points $\sigma_{0} \rightsquigarrow \sigma_{1} \rightsquigarrow \sigma_{2}$ (où $\sigma_{i}$ est le point de codimension $i$ de $\Sigma$ ) et un morphisme $f: \Sigma \rightarrow S$ tel que $f\left(s_{i}\right)=x_{i}$ pour $i=0,1,2$. En faisant le changement de base $\Sigma \rightarrow S$, on se ramène au cas où $S$ est le spectre d'un anneau de valuation de rang 2. Notons $F_{0}$ la fibre spéciale de $F_{P}$, qui est un $S$-schéma de présentation finie. Supposons maintenant que $x_{1} \notin E_{P}$, alors l'image du monomorphisme $F_{0} \rightarrow F_{P} \rightarrow S$ est $\left\{x_{0}, x_{2}\right\}$. Si l'on choisit un trait $T$ et un morphisme $T \rightarrow S$ 
d'image $\left\{x_{0}, x_{2}\right\}$, on voit que la restriction de $F_{P}$ à $T$ est représentable par $T$ lui-même et est donc connexe. Ceci montre que $F_{P}$ et $F_{0}$ sont connexes. D'après le lemme 3.3.5, ceci est exclu. Il s'ensuit que $x_{1} \in E_{P}$ donc $E_{P}$ est ouvert, ce qui conclut la preuve de la proposition.

3.3.3 Lemme. Soit $f: X \rightarrow S$ un monomorphisme de schémas tel que $S$ est un trait et $f(X)$ est le point fermé. Alors $f$ est une immersion fermée.

Preuve : Comme $f$ est un monomorphisme, $X$ est réduit à un point et il est donc affine, d'anneau $A$ artinien. La restriction de $f$ au-dessus du point fermé est un monomorphisme d'un schéma non vide à valeurs dans le spectre d'un corps donc c'est un isomorphisme. D'après le lemme de Nakayama, le morphisme $\Gamma\left(S, \mathcal{O}_{S}\right) \rightarrow A$ est donc surjectif.

3.3.4 Lemme. Soient $s_{0} \rightsquigarrow s_{1} \rightsquigarrow \cdots \rightsquigarrow s_{n}$ des points d'un schéma localement noethérien $S$ tels que $s_{i}$ est une spécialisation de $s_{i-1}$ pour tout $i=1, \ldots, n$. Alors il existe un schéma $T$, spectre d'un anneau de valuation de rang $n$, de points notés $t_{0} \rightsquigarrow t_{1} \rightsquigarrow \cdots \rightsquigarrow t_{n}$ où $t_{i}$ est l'unique point de codimension $i$, et un morphisme $f: T \rightarrow S$ tel que $f\left(t_{i}\right)=s_{i}$ pour tout $i$.

Preuve : On peut remplacer $S$ par l'adhérence de $s_{0}$ puis par son sous-schéma réduit et donc supposer $S$ intègre de corps de fonctions égal au corps résiduel de $s_{0}$. On peut ensuite remplacer $S$ par un ouvert affine contenant $s_{n}$ et on se ramène ainsi au cas où $S$ est le spectre d'un anneau intègre noethérien $A$, de corps de fractions $K=k(S)$. Notons $p_{i} \subset A$ l'idéal premier correspondant au point $s_{i}$ et $A_{p_{i}}$ son anneau local, avec $p_{0}=(0) \subset p_{1} \subset \cdots \subset p_{n} \subset A$ et $A_{p_{n}} \subset \cdots \subset A_{p_{1}} \subset A_{p_{0}}=K$. On considère :

(1) une valuation $v_{1}: K \rightarrow \mathbb{Z}$ dont l'anneau de valuation domine $A_{p_{1}}$. Alors $A_{p_{1}} / p_{1}$ s'identifie à un sous-anneau du corps résiduel $k\left(v_{1}\right)$, de corps de fractions $k\left(v_{1}\right)$;

(2) une valuation $v_{2}: k\left(v_{1}\right) \rightarrow \mathbb{Z}$ dont l'anneau de valuation domine $A_{p_{2}} / p_{1}$. Alors $A_{p_{2}} / p_{2}$ s'identifie à un sous-anneau du corps résiduel $k\left(v_{2}\right)$, de corps de fractions $k\left(v_{2}\right)$;

et ceci jusqu'à :

(n) une valuation $v_{n}: k\left(v_{n-1}\right) \rightarrow \mathbb{Z}$ dont l'anneau de valuation domine $A_{p_{n}} / p_{n-1}$.

On considère alors la valuation lexicographique $v=\left(v_{1}, \ldots, v_{2}\right): K \rightarrow \mathbb{Z}^{n}$ associée aux $v_{i}$ et à un choix d'uniformisantes $\pi_{i} \in k\left(v_{i-1}\right)$ telles que $v_{i}\left(\pi_{i}\right)=1$. Elle est définie précisément ainsi : si $x \in K \backslash\{0\}$, on note $i_{1}:=v_{1}(x)$ et $x_{1}$ la classe résiduelle de $\pi_{1}^{-i_{1}} x$ dans $k\left(v_{1}\right)$, puis $i_{2}:=v_{2}\left(x_{1}\right)$ et $x_{2}$ la classe résiduelle de $\pi_{2}^{-i_{2}} x_{1}$ dans $k\left(v_{2}\right)$, etc. On pose alors $v(x)=\left(i_{1}, \ldots, i_{n}\right)$. On note enfin $V=\{x \in K, v(x) \geq(0, \ldots, 0)\}$ l'anneau de valuation de $v$ et $T=\operatorname{Spec}(V)$. Par construction, on a un morphisme $f: T \rightarrow S$ qui satisfait aux conditions de l'énoncé.

3.3.5 Lemme. Soit $S$ le spectre d'un anneau de valuation de rang 2 et $s_{0} \rightsquigarrow s_{1} \rightsquigarrow s_{2}$ ses points. Alors, il n'existe pas de monomorphisme de présentation finie $f: X \rightarrow S$ tel que $X$ est un schéma connexe et $f(X)=\left\{s_{0}, s_{2}\right\}$.

Preuve : Soit $i$ l'immersion fermée $X_{\text {red }} \hookrightarrow X$, quitte à considérer $f \circ i$ à la place de $f$ on peut supposer que $X$ est réduit. Comme $X$ est connexe, la préimage par $f$ du point fermé $s_{2}$ est un point $y$ fermé dans $X$ mais non ouvert. Il s'ensuit que tout ouvert affine de $X$ contenant $y$ est égal à $X$, 
donc $X$ est affine. La topologie de $X$ est celle d'un trait, en particulier son anneau $B$ est intègre. Soit $V$ l'anneau de valuation dont $S$ est le spectre et $K$ son corps de fractions. La restriction de $X \rightarrow S$ au-dessus du point générique est un monomorphisme d'un schéma non vide à valeurs dans $\operatorname{Spec}(K)$ donc c'est un isomorphisme, donc le corps de fractions de $B$ est $K$. De plus $B$ est sans torsion comme $V$-module, donc plat sur $V$. Comme $X \rightarrow S$ est de présentation finie et plat il est ouvert, contradiction.

3.3.6 Le contre-exemple. L'exemple suivant m'a été suggéré par Angelo Vistoli. Il montre que $\operatorname{Irr}(X / S)^{\mathfrak{f}}$ ne possède pas d'aussi bonnes propriétés de représentabilité que $\pi_{0}(\mathscr{X} / S)^{\mathfrak{f}}$. Sur un corps $k$ de caractéristique différente de 2 , nous considérons l'espace modulaire des coniques planes $S:=$ $\mathbb{P}^{5}=\operatorname{Proj}(k[a, b, c, d, e, f])$ et la conique universelle $X \subset \mathbb{P}^{2} \times \mathbb{P}^{5}$ d'équation $q(x, y, z)=0$ où $q(x, y, z)=a x^{2}+b y^{2}+c z^{2}+d x y+e x z+f y z$. Il y a trois types de coniques:

- les coniques lisses correspondent à l'ouvert $U=\{\operatorname{disc}(q) \neq 0\}$;

- les droites doubles vivent dans le fermé $F$ image du morphisme $\left(\mathbb{P}^{2}\right)^{\vee} \simeq \mathbb{P}^{1} \rightarrow S$ qui envoie une droite d'équation $\ell=0$ sur la conique d'équation $\ell^{2}=0$;

- les coniques singulières réductibles forment la partie localement fermée $(X \backslash U) \backslash F$.

On note que l'ensemble des $s \in S$ tels que $X_{s}$ est géométriquement irréductible n'est pas localement fermé. Posons $F:=\operatorname{Irr}(X / S)^{\mathfrak{f}}$ et montrons que $F$ n'est pas représentable par un $S$ espace algébrique formel. Soit $H \rightarrow S$ le schéma de Hilbert des sous-schémas fermés de $X$ et soit $H_{0} \subset H$ l'ouvert et fermé contenant le sous-schéma fermé maximal $Z=X$. C'est la composante du schéma de Hilbert indicée par le polynôme de Hilbert maximal $P(n)=2 n+1$, et on a $H_{0} \simeq S$. Soit $F_{0} \subset F$ l'ouvert et fermé préimage. Il est clair que $F_{0}$ est le sous-foncteur de $H_{0}$ indicateur du lieu où les fibres de $X \rightarrow S$ sont géométriquement irréductibles. Comme l'ensemble des points $s \in S$ tels que $Z_{s}$ est géométriquement irréductible n'est pas localement fermé, il découle de la proposition 3.3 .1 que $F_{0}$ n'est pas représentable par un $S$-espace algébrique formel. A fortiori, $F$ n'est pas représentable par un $S$-espace algébrique formel.

\subsection{Exemple : modules des courbes admettant une action}

Soit $G$ un groupe fini, $\gamma$ son cardinal et $S=\operatorname{Spec}(\mathbb{Z}[1 / \gamma])$ qui sert de schéma de base. On fixe un entier $g \geq 2$ et on considère le champ $\mathscr{M}_{g}$ des courbes de genre $g$. C'est un champ de Deligne-Mumford lisse et de dimension $3 g-3$.

3.4.1 Proposition. Soit $\mathscr{M}_{g}(G)$ le sous-S-champ de $\mathscr{M}_{g}$ des courbes qui admettent une action fidèle de $G$.

(1) Le champ $\mathscr{M}_{g}(G)$ est un sous-champ fermé, que l'on munit de la structure de sous-champ algébrique réduit. Il est plat, de présentation finie sur $\mathbb{Z}[1 / \gamma]$, à fibres géométriquement réduites.

(2) La normalisation $\widetilde{\mathscr{M}}_{g}(G)$ de $\mathscr{M}_{g}(G)$ est un champ algébrique lisse sur $\mathbb{Z}[1 / 30 \gamma]$.

La preuve utilise le lemme 4.1 de [MSSV] qui montre plus précisément qu'en fait, en dehors d'une liste explicite de 10 groupes, le résultat de (2) vaut aussi sur $\mathbb{Z}[1 / 2 \gamma]$.

Preuve : (1) Notons $\mathscr{H}$ le champ de Hurwitz classifiant les paires $(C, \phi)$ où $C$ est une courbe de genre $g$ et $\phi: G \hookrightarrow \operatorname{Aut}(C)$ est un monomorphisme de schémas en groupes. C'est un champ 
algébrique de Deligne-Mumford lisse sur $\mathbb{Z}[1 / \gamma]$, non équidimensionnel : les dimensions des différentes composantes connexes dépendent de la ramification de l'action du groupe $G$. Le morphisme $f: \mathscr{H} \rightarrow \mathscr{M}_{g}$ donné par l'oubli de l'action est fini (représentable) et non ramifié. Ainsi $\mathscr{M}_{g}(G)$, qui est l'image de $f$, ou encore son image schématique, est fermé. Les composantes irréductibles de $\mathscr{M}_{g}(G)$ sont images de composantes irréductibles de $\mathscr{H}$, et en particulier dominent $\operatorname{Spec}(\mathbb{Z}[1 / \gamma])$. Ceci montre que $\mathscr{M}_{g}(G)$ est plat sur $\mathbb{Z}[1 / \gamma]$. Par ailleurs, comme $f$ est non ramifié, le $\mathcal{O}_{\mathscr{M}_{g}}$-module $f_{*} \mathcal{O}_{\mathscr{H}}$ est localement engendré par un élément, ce qui montre que son annulateur est égal à $\operatorname{Fitt}_{0}\left(f_{*} \mathcal{O}_{\mathscr{H}}\right)$, le 0-ième idéal de Fitting ([Ei], prop. 20.7). De plus, ceci reste vrai après tout changement de base. Comme la formation des idéaux de Fitting commute au changement de base, il en va de même pour l'image (schématique) de $f$. Alors, comme les fibres de $\mathscr{H}$ sur $S$ sont géométriquement réduites, la même chose est vraie pour les fibres de $\mathscr{M}_{g}(G)$.

(2) On peut remplacer $\mathscr{M}_{g}(G)$ par une de ses composantes irréductibles. Notons alors $\eta$ le point générique et $G^{\prime}$ le groupe d'automorphisme de la courbe correspondante; on a donc $G \subset G^{\prime}$ et il est clair que $\mathscr{M}_{g}(G)=\mathscr{M}_{g}\left(G^{\prime}\right)$. De plus on sait d'après [MSSV], lemma 4.1 que les premiers qui divisent $\left[G^{\prime}: G\right]$ sont dans $\{2,3,5\}$ (dans loc. cit., ce résultat est énoncé en caractéristique 0, mais la lecture de la preuve montre que tout est valable en caractéristique première à l'ordre de $G$ ). Ainsi, quitte à se restreindre à $\mathbb{Z}[1 / 30 \gamma]$ et à remplacer $G$ par $G^{\prime}$, on peut supposer que le groupe d'automorphismes de la courbe générique est exactement $G$. Dans la suite, nous supposons cette condition réalisée.

Le champ $\mathscr{H}$ est muni d'une action à gauche du groupe $\operatorname{Aut}(G)$ par 《torsion des actions » définie de la manière suivante : si $\alpha$ est un automorphisme de $G$, on pose $\alpha \cdot(C, \phi)=\left(C, \phi \circ \alpha^{-1}\right)$. Le morphisme $f: \mathscr{H} \rightarrow \mathscr{M}_{g}$ est clairement invariant sous $\operatorname{Aut}(G)$, de sorte qu'il induit un morphisme fini surjectif $f^{\prime}: \mathscr{H} / \operatorname{Aut}(G) \rightarrow \mathscr{M}_{g}(G)$. Comme le groupe d'automorphismes de la courbe générique est $G$, ce morphisme est birationnel. Comme de plus le morphisme de quotient $\mathscr{H} \rightarrow \mathscr{H} / \operatorname{Aut}(G)$ est étale, le champ $\mathscr{H} / \operatorname{Aut}(G)$ est lisse sur $S$ donc normal. D'après le théorème principal de Zariski, $f^{\prime}$ s'identifie à la normalisation de $\mathscr{M}_{g}(G)$.

Dans la terminologie en vigueur, ce résultat montre que $\mathscr{M}_{g}(G)$ est équinormalisable, et même équidésingularisable :

3.4.2 Corollaire. Soit $S=\operatorname{Spec}(\mathbb{Z}[1 / 30 \gamma])$ et $S^{\prime} \rightarrow S$ un changement de base avec $S^{\prime}$ normal. Alors $\widetilde{\mathscr{M}_{g}}(G) \times_{S} S^{\prime}$ est la normalisation de $\mathscr{M}_{g}(G) \times_{S} S^{\prime}$. Les fibres de $\widetilde{\mathscr{M}_{g}}(G) \rightarrow S$ sont les normalisations des fibres de $\mathscr{M}_{g}(G)$.

Preuve : Notons d'abord que $\mathscr{M}_{g}(G) \times_{S} S^{\prime}$ (resp. $\widetilde{\mathscr{M}_{g}}(G) \times_{S} S^{\prime}$ ) est à fibres géométriquement réduites (resp. est lisse) sur $S^{\prime}$ normal, donc il est réduit (resp. normal). Le sous-champ ouvert $\mathscr{U} \subset \mathscr{M}_{g}(G)$ au-dessus duquel le morphisme $f^{\prime}: \mathscr{H} / \operatorname{Aut}(G) \rightarrow \mathscr{M}_{g}(G)$ est plat sur $S$ et dense fibre à fibre, donc $S$-universellement dense ([EGA] IV.11.10.9). Il s'ensuit que le morphisme $\widetilde{\mathscr{M}}_{g}(G) \times_{S}$ $S^{\prime} \rightarrow \mathscr{M}_{g}(G) \times{ }_{S} S^{\prime}$ est un isomorphisme au-dessus de $\mathscr{U} \times{ }_{S} S^{\prime}$, donc birationnel. Ce morphisme est aussi quasi-fini, séparé, surjectif, donc c'est la normalisation de $\mathscr{M}_{g}(G) \times_{S} S^{\prime}$.

3.4.3 Corollaire. Soit $S=\operatorname{Spec}(\mathbb{Z}[1 / 30 \gamma])$. Le foncteur $\operatorname{Irr}\left(\mathscr{M}_{g}(G) / S\right)$ est représentable par un S-schéma fini étale.

Preuve : Puisque la normalisation $\pi: \widetilde{\mathscr{M}}_{g}(G) \rightarrow \mathscr{M}_{g}(G)$ est $S$-birationnelle, elle induit un isomorphisme $\operatorname{Irr}\left(\widetilde{\mathscr{M}_{g}}(G) / S\right) \simeq \operatorname{Irr}\left(\mathscr{M}_{g}(G) / S\right.$ ) (corollaire 2.6.2). Comme de plus $\widetilde{\mathscr{M}_{g}}(G) / S$ est normal sur $S$, on a bien sûr $\operatorname{Irr}\left(\widetilde{\mathscr{M}}_{g}(G) / S\right)=\pi_{0}\left(\widetilde{\mathscr{M}}_{g}(G) / S\right)$. Pour finir, il est connu que le champ 
$\widetilde{\mathscr{M}_{g}}(G)=\mathscr{H} / \operatorname{Aut}(G)$ admet une compactification lisse $\overline{\mathscr{H}}$ dans laquelle il est $S$-dense (voir [BR], section 6.3). Il en découle que $\pi_{0}\left(\widetilde{\mathscr{M}}_{g}(G) / S\right)$ est isomorphe à $\pi_{0}(\overline{\mathscr{H}} / S)$ et ce dernier foncteur est représentable par un schéma fini étale, d'après la proposition 3.2 .5 .

\section{A Propriétés constructibles pour les champs algébriques}

Dans cette annexe, nous rappelons quelques résultats de [EGA] IV concernant la constructiblité de certaines parties dans des champs algébriques. Les démonstrations, écrites dans le cas des schémas, s'adaptent de manière à peu près immédiate au cas des morphismes de champs algébriques, pourvu que l'on soit suffisamment soigneux dans les énoncés. En général, les adaptations nécessaires reviennent à remplacer, lorsque nécessaire, l'utilisation de recouvrements par des schémas affines ouverts de Zariski par des recouvrements par des schémas affines ouverts pour la topologie lisse; ou alors à appliquer les résultats de [EGA] pour des groupoïdes $X_{1} \rightrightarrows X_{0}$, c'est-à-dire des paires de morphismes satisfaisant certaines conditions, au lieu de les appliquer simplement pour des schémas; ou à utiliser d'autres techniques du même genre, classiques lorsqu'on manipule des champs algébriques.

Ci-dessous, nous réunissons un ensemble de résultats qui incluent d'une part ceux dont nous avons besoin dans le corps de l'article, et d'autre part ceux qui sont nécessaires à la preuve (adaptée de [EGA IV) des précédents. Après chaque énoncé, nous indiquons chaque fois que cela est utile les modifications à apporter à la preuve de [EGA] pour passer des schémas aux champs algébriques.

A.1 Fibres des morphismes de champs algébriques. Soit $f: \mathscr{X} \rightarrow \mathscr{S}$ un morphisme de champs algébriques et $s \in|\mathscr{S}|$ un point. Même lorsque $s$ possède un corps résiduel $k(s)$ bien défini, il n'existe pas en général de morphisme $\operatorname{Spec}(k(s)) \rightarrow \mathscr{S}$, de sorte que la notion de fibre de $f$ au point $s$ n'est pas aussi immédiate que dans le cas des morphismes de schémas. Pour prendre ce fait en compte, nous utiliserons la convention de terminologie suivante.

Considérons une propriété de la forme $P=P(\mathscr{X} / k, \mathscr{F}, Z)$ portant sur des données composées d'un champ algébrique $\mathscr{X}$ sur un corps $k$, un $\mathcal{O}_{\mathscr{X}}$-module $\mathscr{F}$ et une partie $Z \subset|\mathscr{X}|$. (De la même façon, les considérations qui suivent sont valables pour des propriétés mettant en jeu un nombre fini de champs algébriques, de modules ou de parties sur ces champs, de morphismes entre ces champs... ou une partie seulement de ces données.) On s'intéressera principalement à des propriétés indépendantes du corps de base au sens où, pour toute extension de corps $\ell / k, P(\mathscr{X} / k, \mathscr{F}, Z)$ est vraie si et seulement si $P\left(\mathscr{X}_{\ell} / \ell, \mathscr{F}_{\ell}, Z_{\ell}\right)$ est vraie.

Une telle propriété $P$ étant fixée, revenons à un morphisme de champs algébriques $f: \mathscr{X} \rightarrow \mathscr{S}$ et un point $s=\left[s_{K}\right]$. Pour tout représentant $s_{K}: \operatorname{Spec}(K) \rightarrow \mathscr{S}$ de $s$, où $K$ est un corps, on note $\mathscr{X}_{K}=\mathscr{X} \times \mathscr{S} \operatorname{Spec}(K), \mathscr{F}_{K}$ la préimage de $\mathscr{F}$ par la projection $\mathscr{X}_{K} \rightarrow \mathscr{X}, Z_{K}$ la préimage de $Z$ par l'application continue $\left|\mathscr{X}_{K}\right| \rightarrow|\mathscr{X}|$. Compte tenu de l'hypothèse sur $P$, le fait que $P\left(\mathscr{X}_{K} / K, \mathscr{F}_{K}, Z_{K}\right)$ ait lieu est indépendant du représentant $s_{K}: \operatorname{Spec}(K) \rightarrow \mathscr{S}$ choisi pour $s$. On dira alors que la propriété $P\left(\mathscr{X}_{s}, \mathscr{F}_{s}, Z_{s}\right)$ est vraie. On notera qu'il s'agit bien sûr d'un abus de langage, puisque nous n'avons défini ni $\mathscr{X}_{s}$, ni $\mathscr{F}_{s}$, ni $Z_{s}$. À chaque fois que nous utiliserons cette notation $P\left(\mathscr{X}_{s}, \mathscr{F}_{s}, Z_{s}\right)$, il sera sous-entendu que la propriété $P$ est indépendante du corps de base au sens ci-dessus (et cette indépendance sera évidente ou bien connue).

A.2 Propriétés constructibles. Dans les énoncés ci-dessous, la vérification du fait qu'une propriété $P$ ait lieu se ramène toujours au cas où $\mathscr{S}$ est un schéma, après changement de base par une présentation lisse $S \rightarrow \mathscr{S}$. 
Concernant la constructibilité, on notera que si $\mathscr{X}$ est un champ algébrique et $Z$ est une partie de $|\mathscr{X}|$, la propriété pour $Z$ d'être constructible est locale sur $\mathscr{X}$ pour la topologie lisse. On a même une propriété beaucoup plus forte, puisque $Z$ est constructible si et seulement si $u^{-1}(Z)$ est constructible, pour n'importe quel morphisme surjectif et ouvert $u: \mathscr{U} \rightarrow \mathscr{X}$.

A.2.1 Théorème (Chevalley) Soit $f: \mathscr{X} \rightarrow \mathscr{S}$ un morphisme de présentation finie de champs algébriques et soit $Z$ une partie constructible de $|\mathscr{X}|$. Alors $f(Z)$ est une partie constructible de $|\mathscr{S}|$.

Voir [LMB], théorème 5.9.4.

A.2.2 Proposition. Soient $\mathscr{X} \rightarrow \mathscr{S}$ un morphisme de présentation finie de champs algébriques et $Z, Z^{\prime}$ deux parties localement constructibles de $|\mathscr{X}|$. Alors, les ensembles suivants sont localement constructibles dans $|\mathscr{S}|$ :

(i) l'ensemble des $s \in|\mathscr{S}|$ tels que $Z_{s} \neq \emptyset$,

(ii) l'ensemble des $s \in|\mathscr{S}|$ tels que $Z_{s} \subset Z_{s}^{\prime}$ (resp. $Z_{s}=Z_{s}^{\prime}$ ),

(iii) si $Z \subset Z^{\prime}$, l'ensemble des $s \in|\mathscr{S}|$ tels que $Z_{s}$ est dense dans $Z_{s}^{\prime}$,

(iv) l'ensemble des $s \in|\mathscr{S}|$ tels que $Z_{s}$ est ouvert (resp. fermé, resp. localement fermé) dans $\left|\mathscr{X}_{s}\right|$.

Voir [EGA] IV.9.5.1, 9.5.2, 9.5.3, 9.5.4. Les preuves de 9.5.1, 9.5.2 et de 9.5.4 (une fois démontré 9.5.3) s'adaptent immédiatement au cas des champs algébriques. La preuve de 9.5.3 pour un morphisme de schémas $f: X \rightarrow S$ se ramène au cas où $X$ est intègre. Ensuite, on utilise un recouvrement de $X$ par des ouverts affines intègres. Dans le cas des champs algébriques, l'utilisation d'un recouvrement lisse de $\mathscr{X}$ par des ouverts affines intègres fait tout aussi bien l'affaire.

A.2.3 Proposition. Soient $\mathscr{X} \rightarrow \mathscr{S}, \mathscr{Y} \rightarrow \mathscr{S}$ deux morphismes de présentation finie de champs algébriques et $f: \mathscr{X} \rightarrow \mathscr{Y}$ un $\mathscr{S}$-morphisme. Alors, les ensembles suivants sont localement constructibles dans $|\mathscr{S}|$ :

(i) l'ensemble des $s \in|\mathscr{S}|$ tels que $f_{s}$ est une immersion,

(ii) l'ensemble des $s \in|\mathscr{S}|$ tels que $f_{s}$ est une immersion fermée,

(iii) l'ensemble des $s \in|\mathscr{S}|$ tels que $f_{s}$ est une immersion ouverte.

Voir [EGA] IV.9.6.1, (viii), (ix), (x).

La proposition suivante fait intervenir la notion de point associé d'un faisceau quasi-cohérent sur un champ algébrique. La définition correcte est la suivante; elle est tirée du paragraphe 2.2.6.3 de Lieblich [Lie], auquel nous renvoyons le lecteur pour plus de détails.

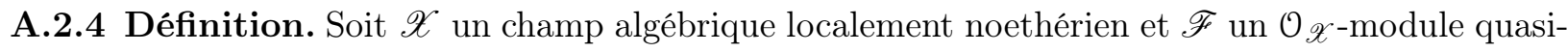
cohérent. On dit qu'un point $x \in|\mathscr{X}|$ est un point associé de $\mathscr{F}$ s'il existe un sous-faisceau $\mathscr{G} \subset \mathscr{F}$ quasi-cohérent tel que $x \in \operatorname{Supp}(\mathscr{G}) \subset \overline{\{x\}}$. On note $\operatorname{Ass}(\mathscr{F})$ l'ensemble des points associés de $\mathscr{F}$.

A.2.5 Proposition. Soient $\mathscr{S}$ un champ algébrique noethérien intègre de point générique $\eta, f$ : $\mathscr{X} \rightarrow \mathscr{S}$ un morphisme de type fini, $\mathscr{F}$ un $\mathcal{O}_{\mathscr{X}}$-module cohérent. Si $\mathscr{F}_{\eta}$ est sans cycle premier associé immergé, il existe un voisinage $\mathscr{U}$ de $\eta$ dans $\mathscr{S}$ tel que pour tout $s \in|\mathscr{U}|$, $\mathscr{F}_{s}$ soit sans cycle premier associé immergé. 
Voir [EGA] IV .9.7.6. Le cas des champs algébriques en découle en prenant une présentation lisse $\pi: X \rightarrow \mathscr{X}$, car $\mathscr{F}$ est sans cycle premier associé immergé si et seulement si $\pi^{*} \mathscr{F}$ est sans cycle premier associé immergé.

A.2.6 Théorème. Soit $\mathscr{X} \rightarrow \mathscr{S}$ un morphisme de présentation finie de champs algébriques. Alors, les ensembles suivants sont localement constructibles dans $|\mathscr{S}|$ :

(i) l'ensemble des $s \in|\mathscr{S}|$ tels que $\mathscr{X}_{s}$ est géométriquement irréductible,

(ii) l'ensemble des $s \in|\mathscr{S}|$ tels que $\mathscr{X}_{s}$ est géométriquement connexe,

(iii) l'ensemble des $s \in|\mathscr{S}|$ tels que $\mathscr{X}_{s}$ est géométriquement réduit,

(iv) l'ensemble des $s \in|\mathscr{S}|$ tels que $\mathscr{X}_{s}$ est géométriquement intègre.

Voir [EGA] IV .9.7.7. Pour adapter la démonstration au cas des champs algébriques, on se ramène immédiatement au cas où $\mathscr{S}$ est un schéma $S$ en prenant les images inverses par une présentation lisse $S \rightarrow \mathscr{S}$.

Le principal point délicat se situe alors dans le numéro $1^{\circ}$ de loc. cit. où l'on construit un ouvert $W$ commun à $X$ et à un schéma de la forme $Y=\operatorname{Spec}\left(A\left[T_{1}, \ldots, T_{n+1}\right] /(F)\right)$ (notations de loc. cit.). Lorsque $\mathscr{X}$ est un champ algébrique, nous procéderons comme suit. On considère une présentation lisse quasi-compacte $X_{0, \eta} \rightarrow \mathscr{X}_{\eta}$ de la fibre de $\mathscr{X}$ au-dessus du point générique de $S$, et on pose $X_{1, \eta}=X_{0, \eta} \times \mathscr{X} X_{0, \eta}$. On note $\delta_{0, i}\left(1 \leq i \leq n_{0}\right)$ (resp. $\left.\delta_{1, j} 1 \leq j \leq n_{1}\right)$ les points génériques de $X_{0, \eta}$ (resp. de $X_{1, \eta}$ ) et $L_{0, i}$ (resp. $L_{1, j}$ ) leurs anneaux locaux, qui sont des corps. On « déploie » chacun des corps de fonctions $L_{a, i}$ en un schéma affine de la forme $Y_{a, i}=\operatorname{Spec}\left(A\left[T_{a, i, 1}, \ldots, T_{a, i, n+1}\right] /\left(F_{a, i}\right)\right)$ par le procédé de EGA. Notons $Y_{0}$ resp. $Y_{1}$ le schéma somme disjointe des $Y_{0, i}$ resp. des $Y_{1, i}$. Par construction $Y_{0, \eta}$ resp. $Y_{1, \eta}$ est somme de schémas intègres de corps de fonctions rationnelles les $L_{a, i}$, et on dispose donc de deux flèches $Y_{1, \eta} \rightarrow Y_{0, \eta}$ définies en codimension 0 (ce sont les restrictions des deux flèches $X_{1, \eta} \rightarrow X_{0, \eta}$ aux points génériques). Comme $Y_{0}, Y_{1}$ sont de présentation finie, quitte à les remplacer par des ouverts, on peut supposer que ces deux flèches s'étendent en des morphismes $f, g: Y_{1} \rightarrow Y_{0}$. Comme au-dessus de $\eta$ ces morphismes coïncident génériquement avec les deux flèches $X_{1, \eta} \rightarrow X_{0, \eta}$ qui sont lisses, quitte à restreindre encore $Y_{0}$ et $Y_{1}$ on peut supposer que $f, g$ sont lisses. Elles définissent donc un groupoïde lisse dont on note $\mathscr{Y}$ le champ algébrique quotient. Ce champ algébrique joue le rôle tenu par $Y$ dans la preuve de [EGA] IV.9.7.7.

On doit ensuite justifier qu'il existe un voisinage ouvert $U$ de $\eta$ dans $S$ tel que $\mathscr{Y}_{s}$ reste géométriquement intègre pour tout $s \in U$. Or $\mathscr{Y}_{s}$ est géométriquement intègre si et seulement si $Y_{0, s}$ est géométriquement ponctuellement intègre et $Y_{1, s} \rightarrow Y_{0, s} \times Y_{0, s}$ est dominant. On obtient donc l'existence d'un tel $U$ en utilisant [EGA] IV.9.7.4, 9.7.5 et 9.6.1(ii).

A.2.7 Proposition. Soit $\mathscr{X} \rightarrow \mathscr{S}$ un morphisme de présentation finie de champs algébriques. Alors les fonctions "nombre géométrique de composantes connexes de $\mathscr{X}_{s}$ » et «nombre géométrique de composantes irréductibles de $\mathscr{X}_{s} \gg$ sont localement constructibles.

Voir [EGA IV.9.7.9. La preuve utilise IV.9.7.8 et IV.9.7.1, dont les énoncés et les preuves s'adaptent sans modification pour les champs algébriques. Pour ne pas alourdir inutilement le texte, nous ne les recopions pas ici.

\section{B Pureté pour les champs algébriques}

Si $X \rightarrow S$ est un morphisme de schémas localement de type fini et $\mathscr{M}$ est un $\mathcal{O}_{X}$-module quasi-cohérent de présentation finie, la notion de pureté de $\mathscr{M}$ relativement à $S$ est définie dans 
[RG], 3.3.3. Si $\mathscr{M}$ est plat sur $S$, cette définition est locale pour la topologie plate sur $S$ ([RG], 3.3.7). En revanche, elle n'est pas locale sur $X$, même pour la topologie de Zariski. En conséquence, l'extension de cette notion au cas où $\mathscr{X} \rightarrow \mathscr{S}$ est un morphisme de champs algébriques ne peut se faire simplement en prenant une présentation lisse de $\mathscr{X}$. On en revient donc à la définition originale, passant par les points associés (définition A.2.4).

B.1 Définition. Soit $f: \mathscr{X} \rightarrow \mathscr{S}$ un morphisme localement de type fini de champs algébriques et soit $\mathscr{M}$ un $\mathcal{O}_{\mathscr{X}}$-module quasi-cohérent de présentation finie, plat sur $\mathscr{S}$.

(i) Supposons que $\mathscr{S}$ est un schéma local hensélien $S$ de point fermé $s_{0}$. On dit que $\mathscr{M}$ est pur relativement à $S$ si pour tout $x \in|\mathscr{X}|$, qui est un point associé du $\mathcal{O}_{\mathscr{X}_{s}}$-module $\mathscr{M}_{s}$, où $s=f(x)$, l'adhérence de $x$ dans $|\mathscr{X}|$ rencontre $\mathscr{X}_{s_{0}}$.

(ii) Supposons que $\mathscr{S}$ est représentable par un espace algébrique $S$. On dit que $\mathscr{M}$ est pur relativement à $S$ si pour tout $s \in S$, de hensélisé $\left(S^{h}, s^{h}\right)$, le module $\mathscr{M} \times{ }_{S} S^{h}$ est pur relativement à $S^{h}$.

(iii) En général, on dit que $\mathscr{M}$ est pur relativement à $\mathscr{S}$ si $\pi^{*} \mathscr{M}$ est pur relativement à $S$, pour une (et donc toute) présentation lisse $\pi: S \rightarrow \mathscr{S}$.

B.2 Lemme. Soit $R=(R, K, k, \pi)$ un anneau de valuation discrète et $\mathscr{X}$ un $R$-champ algébrique localement de type fini, plat, à fibre spéciale réduite. Alors, $\mathscr{X}$ est connexe si et seulement si $\mathscr{X}_{K}$ est connexe, et $\mathscr{X}$ est intègre si et seulement si $\mathscr{X}_{K}$ est intègre.

Preuve : Posons $B=H^{0}\left(\mathscr{X}, \mathcal{O}_{\mathscr{X}}\right)$, on a $B_{K}=H^{0}\left(\mathscr{X}_{K}, \mathcal{O}_{\mathscr{X}_{K}}\right)$. Pour la première assertion, il suffit de montrer que les idempotents de $B$ et $B_{K}$ sont les mêmes, et donc de montrer que les idempotents de $B_{K}$ sont dans $B$. Soit $e \in B_{K}$ tel que $e^{2}=e$. Si $e \notin B$, il existe une écriture $e=\pi^{-n} f$ avec $n \geq 1$ et $f \in B \backslash \pi B$. Comme $f^{2}=\left(\pi^{n} e\right)^{2}=\pi^{n} f$ et que $B_{k}$, en tant que sous-anneau de $H^{0}\left(\mathscr{X}_{k}, \mathcal{O}_{\mathscr{X}_{k}}\right)$, est réduit par hypothèse, on trouve que $f$ est nul modulo $\pi$, contradiction. Pour la seconde assertion, il ne reste qu'à montrer que $\mathscr{X}$ est localement intègre si et seulement si $\mathscr{X}_{K}$ l'est. Or par platitude cela est clair si $\mathscr{X}$ est un schéma, et on se ramène à ce cas en utilisant une présentation lisse $X \rightarrow \mathscr{X}$.

B.3 Lemme. Soit $R$ un anneau de valuation discrète hensélien et $\mathscr{X}$ un $R$-champ algébrique de type fini, plat et pur. Alors, il existe un $R$-schéma affine $U$ avec une $R$-algèbre de fonctions de type fini, libre comme $R$-module, et un morphisme lisse $R$-universellement schématiquement dominant $U \rightarrow \mathscr{X}$. En particulier $H^{0}\left(\mathscr{X}, \mathcal{O}_{\mathscr{X}}\right)$ est un $R$-module libre. Si $\mathscr{X}$ est à fibre spéciale irréductible, on peut supposer que $U$ est réunion disjointe d'un nombre fini d'ouverts affines à fibre spéciale irréductible et algèbre de fonctions de type fini libre comme R-module.

Preuve : Fixons une présentation lisse $X \rightarrow \mathscr{X}$. En chaque point $x \in X$ de la fibre spéciale, choisissons un voisinage ouvert affine $U_{x}$. Si $\mathscr{X}_{k}$ est irréductible, on peut choisir $U_{x}$ à fibre spéciale irréductible. Quitte à rétrécir $U_{x}$, on peut supposer de plus que son anneau de fonctions est séparé pour la topologie $\pi$-adique (voir [Ro2], lemma 2.1.11). D'après Raynaud et Gruson, un tel anneau est libre comme $R$-module (voir [Ro2], lemma 2.1.7). Comme $\mathscr{X}$ (et donc aussi $\mathscr{X}_{k}$ ) est quasi-compact, un nombre fini des ouverts $U_{x}$ recouvre $\mathscr{X}_{k}$. Soit $U$ la somme disjointe de ces ouverts. Comme $\mathscr{X}$ est pur, aucun cycle premier associé de $\mathscr{X}$ n'est inclus dans $\mathscr{X}_{K}$. Un tel cycle premier associé est alors inclus dans l'image de $U$, de sorte que le morphisme $U \rightarrow \mathscr{X}$ est schématiquement dominant. Comme $U_{k} \rightarrow \mathscr{X}_{k}$ l'est aussi, il s'ensuit que $U \rightarrow \mathscr{X}$ est universellement schématiquement dominant (par l'argument de [Ro2], lemma 2.1.9). Il s'ensuit qu'on a une injection $H^{0}\left(\mathscr{X}, \mathcal{O}_{\mathscr{X}}\right) \hookrightarrow H^{0}\left(U, \mathcal{O}_{U}\right)$. 
Comme un sous-module libre d'un module libre sur un anneau de valuation discrète est libre, ceci prouve le lemme.

Le théorème 2.2.1 de [Ro2] se généralise immédiatement:

B.4 Théorème. Soit $f: \mathscr{X} \rightarrow \mathscr{S}$ un morphisme de champs algébriques de présentation finie, plat et pur, et soit $n \geq 1$ un entier. Alors, les ensembles suivants sont ouverts dans $|\mathscr{S}|$ :

(i) l'ensemble des $s \in|\mathscr{S}|$ tels que $\mathscr{X}_{s}$ est géométriquement réduit,

(ii) l'ensemble des $s \in|\mathscr{S}|$ tels que la fibre géométrique $\mathscr{X}_{\bar{s}}$ est réduite avec au plus $n$ composantes connexes,

(iii) l'ensemble des $s \in|\mathscr{S}|$ tels que la fibre géométrique $\mathscr{X}_{\bar{s}}$ est réduite avec au plus $n$ composantes irréductibles.

Preuve : On adapte la preuve de [Ro2], th.2.2.1. En faisant le changement de base par une présentation lisse $S \rightarrow \mathscr{S}$, on se ramène au cas où $\mathscr{S}$ est un schéma $S$. On se ramène ensuite au cas où $S$ est affine, noethérien. D'après A.2.6 (iii) et A.2.7, les ensembles qui nous intéressent sont constructibles dans $S$. On se ramène alors au cas où $S$ est le spectre d'un anneau de valuation discrète $R=(R, K, k, \pi)$, complet à corps résiduel algébriquement clos, puis, en prenant la clôture intégrale de $R$ dans une extension finie de $K$, on se ramène à prouver que $\mathscr{X}_{K}$ est réduit (resp. possède au plus $n$ composantes connexes, resp. possède au plus $n$ composantes irréducitbles) dès que $\mathscr{X}_{k}$ possède la même propriété.

(i) On prouve que $\mathscr{X}_{K}$ est réduit exactement comme dans [Ro2], th. 2.2.1, en remplaçant l'ouvert universellement schématiquement dominant $U$ réunion d'ouverts affines purs utilisé dans loc. cit. par le morphisme $U \rightarrow \mathscr{X}$ fourni par le lemme B.3.

(ii) La preuve de [Ro2, th. 2.2.1 est valable sans modification.

(iii) Le début de la preuve de [Ro2], th. 2.2.1 est valable sans modification, jusqu'au moment où l'on fait appel à un recouvrement ouvert schématiquement dominant par des affines purs. On remplace le recours à ce recouvrement par l'utilisation du morphisme $U \rightarrow \mathscr{X}$ fourni par le lemme B.3, où $U$ est somme disjointe d'ouverts affines $U_{x}$ à fibre spéciale intègre et d'anneau de fonction libre comme $R$-module.

\section{Références}

[AOV] D. Abramovich, M. Olsson, A. Vistoli, Tame stacks in positive characteristic, Ann. Inst. Fourier (Grenoble) 58 (2008), no. 4, 1057-1091.

[Ar1] M. Artin, Versal deformations and algebraic stacks, Invent. Math. 27 (1974), 165-189.

[BR] J. Bertin, M. Romagny, Champs de Hurwitz, prépublication disponible à l'adresse http ://people.math.jussieu.fr/ romagny/.

[Bro] S. Brochard, Foncteur de Picard d'un champ algébrique, Math. Ann. 343 (2009), 541-602.

[DM] P. Deligne, D. Mumford, The irreducibility of the space of curves of given genus, Publ. Math. IHÉS 36 (1969), 75-109.

[EGA] J. Dieudonné, A. Grothendieck, Éléments de Géométrie Algébrique II, III, IV, Publ. Math. IHÉS 8 (1961), 17 (1963), 24 (1965), 28 (1966), 32 (1967). 
[Ei] D. Eisenbud, Commutative algebra with a view toward algebraic geometry, Graduate Texts in Math., Springer-Verlag (1995).

[Kn] D. Knutson, Algebraic spaces, Lecture Notes in Mathematics, Vol. 203, Springer-Verlag, 1971.

[La] Y. LASZLO, Linearization of group stack actions and the Picard group of the moduli of $\mathrm{SL}_{r} / \mu_{s}-$ bundles on a curve, Bull. Soc. Math. France 125 (1997), no. 4, 529-545.

[Lie] M. Lieblich, Moduli of twisted sheaves, Duke Math. J. 138 (2007), no. 1, 23-118.

[LMB] G. Laumon, L. Moret-Bailly, Champs algébriques, Ergebnisse der Mathematik und ihrer Grenzgebiete (3. Folge) no 39, Springer-Verlag, Berlin, 2000.

[MSSV] K. Magaard, T. Shaska, S. Shpectorov, H. Völklein, The locus of curves with prescribed automorphism group, Communications in arithmetic fundamental groups (Kyoto, 1999/2001). Sūrikaisekikenkyūsho Kōkyūroku no. 1267 (2002), 112-141.

[Ol] M. Olsson, On proper coverings of Artin stacks, Adv. Math. 198 (2005), no. 1, 93-106.

[RG] M. Raynaud, L. Gruson, Critères de platitude et de projectivité. Techniques de "platification » d'un module, Invent. Math. 13 (1971), 1-89.

[Ro1] M. Romagny, Group actions on stacks and applications, Michigan Math. J. 53 (2005), no. 1, 209-236.

[Ro2] M. Romagny, Effective models of group schemes, http ://arxiv.org/abs/0904.3167.

[TV] B. ToËn, G. Vezzosi, Homotopical algebraic geometry II. Geometric stacks and applications, Mem. Amer. Math. Soc. 193 (2008), no. 902, x+224 pp. 